\title{
Performance Analysis of Deterministic Key Allocation Schemes for Wireless Sensor Networks
}

\author{
Abhishek Gupta \\ SAIT India Lab., \\ Samsung India Software Operations, \\ Bangalore 560093, India \\ guptaa80@gmail.com \\ Joy Kuri \\ Centre for Electronics Design and Technology \\ Indian Institute of Science \\ Bangalore 560012, India \\ kuri@cedt.iisc.ernet.in \\ Sugata Sanyal \\ School of Technology \& Computer Science \\ Tata Institute of Fundamental Research \\ Homi Bhabha Road, Mumbai 400005, India \\ sanyal@tifr.res.in
}

\begin{abstract}
Abhishek Gupta:
Abhishek Gupta is currently working for a networking group at SAIT India Lab, Samsung, Bangalore. He received his B. Tech. in Electrical Engineering from Institute of Technology, Banaras Hindu University in 2004 and his M. Sc (Engg) in wireless communication from Indian Institute of Science in 2007. His research interests include communication networks, wireless communication and computer algorithms.
\end{abstract}

\section{Joy Kuri:}

Joy Kuri has a B.E. in Electronics and Telecommunication Engineering from Jadavpur University, Kolkata, India. After a year in the industry, he joined the M.E. program in the Department of Electrical Communication Engineering at the Indian Institute of Science. He went on to receive a $\mathrm{PhD}$ from the same department at IISc in 1995. Subsequently, he spent two years at Ecole Polytechnique, University of Montreal, Canada and one and a half years in INRS-Telecommunications, University of Quebec, Canada as a Research Associate.

Since 1999 he has been with the Centre for Electronics Design and Technology, Indian Institute of Science, where he is currently Professor. He is a co-author of the books "Communication Networking: An Analytical Approach" and "Wireless Networking," both published by Morgan Kaufmann, an imprint of Elsevier. He has over 75 publications in international journals and conferences. His research and teaching interests are in the areas of modelling, analysis and control of communication networks and stochastic systems.

\section{Sugata Sanyal: \\ Sugata Sanyal is a Professor in the School of Technology \& Computer Science at the Tata Institute of Fundamental Research (http://www.tifr.res.in/ sanyal). In the early phase of his career, Sugata was involved in huge developmental projects, designing Rugged Micro programmed Computers and Fault-Tolerant Computerized systems. He has worked in diverse areas of Computer Architecture, Parallel Processing, Fault Tolerance and Coding Theory and in the area of Security. Sanyal is in the Editorial Board of many International Journals, and is collaborating with scientists from India and abroad.}


Abstract-Because of the resource-constrained nature of Wireless Sensor Networks (WSNs), it is easier to use a security system based on symmetric key cryptography. However, the distribution and management of cryptographic keys pose challenges of their own. In this paper, we study some deterministic methods of distribution of keys to nodes. We propose and analyze a polynomial-based scheme and a complete connectivity scheme. An analytical comparison between the schemes, in terms of security and connectivity, is presented next. Motivated by the schemes, we present a general key allocation scheme and derive general expressions for security and connectivity that are applicable to any deterministic key distribution algorithm in a certain class (not just the polynomial and full-connectivity schemes mentioned above). With the help of these expressions, we present a number of trade-offs which should be taken into account while designing a secure system.

\section{INTRODUCTION}

Recent advances in wireless communication have opened interesting and challenging areas for research. Wireless sensor networks [Akyildiz et al., 2002], [Culler et al., 2004] is one such field of research. These networks are formed by cheap and highly resource constrained sensor nodes, generally called as motes. The size of the network may vary from few hundreds of nodes to thousands, depending on the type of application for which they are being used.

A typical sensor node contains some sensors (light, temperature, acceleration etc.), a radio chipset for wireless communication, an EEPROM chip for logging sensor data, a node-to-host communication interface (typically a serial port), and a micro-controller which contains some amount of flash memory for program storage and RAM for program execution [Becher et al., 2006].

Wireless sensor networks find numerous applications in many diverse areas. Some of these application areas include environmental and habitat monitoring [Szewczyk et al., 2004], battle field surveillance and border monitoring [Bokareval et al., 2006], health care applications and monitoring of vehicular movements in cities and highways [Akyildiz et al., 2002]. When sensor networks are deployed in hostile environments, some of the applications require communication among nodes and between nodes and base station to be secure. For example, in a surveillance application [Chong and Kumar, 2003], it would be very undesirable if intruders can access the information being carried by the network.

Since sensor networks are adhoc networks with no preexisting infrastructure, public key cryptography may be difficult to implement. Due to the resource constrained nature of the sensors, implementations of public key cryptographic primitives turn out to be costly. Although recent advances have shown the feasibility of public key algorithms in sensor networks [Wang and Li, 2006], [Batina et al., 2006], [Uhsadel et al., 2007], it seems that frequent use of such systems could still make them expensive in terms of computational time and energy consumption. The alternative solution based on symmetric key cryptography is easier to implement. However, the distribution and management of cryptographic keys pose challenges. One requires efficient key distribution algorithms for such type of networks.

A number of key distribution algorithms have been proposed in the literature which require pre-distributing some keys to each node from a given pool of keys. These algorithms can be split into two broad categories, based on the connectivity of the resulting key graph. By a key graph, we mean a graph $\mathcal{G}(V, E)$ where $V$ is the set of vertices corresponding to nodes in the network and $E$ is the set of edges where an edge exists between two vertices only if the corresponding nodes share at least one key.

- Partial connectivity schemes: The keys are distributed in such a fashion that the resulting key graph is partially connected. This means that some of the nodes share keys in common and some do not. Those which do not share a key in common, undertake pairwise key distribution. As will be shown later, such type of schemes show "good" security properties.

- Complete connectivity schemes: The keys are distributed in such a fashion that the resulting key graph is fully connected and does not require any pairwise key setup phase, leading to energy saving. Such type of schemes find applications in areas where sensor life-time is an important factor for design consideration and security comes only as a secondary design criterion.

Let $\mathcal{P}$ be the key pool size and $S_{i}(i=1,2, \ldots n, n$ being the total number of nodes in the network) be the set of keys (called "key ring") assigned to node $i$. That is, $S_{i}=\left\{k_{1}^{i}, k_{2}^{i}, \ldots, k_{k}^{i}\right\}$ where $k$ is the key ring size. Also, let $S_{n}^{k}=\left\{S_{1}, S_{2}, \ldots, S_{n}\right\}$ be the set of key rings corresponding to a network of $n$ nodes where $\left|S_{i}\right|=k \forall$ $i=1,2, \ldots n,\left|S_{n}^{k}\right|=n$ and $\left|\bigcup_{i=1}^{n} S_{i}\right|=\mathcal{P}$. Then, the partial connectivity key distribution schemes satisfy the property $\operatorname{Pr}\left(S_{i} \cap S_{j}=\phi\right)>0$ for $i \neq j$ or $S_{i} \cap S_{j}=\phi$, for some $i$ and $j$ where $i \neq j$. The complete connectivity key distribution schemes satisfy the property $S_{i} \cap S_{j} \neq \phi$ $\forall S_{i}, S_{j} \in S_{n}^{k}$ and $\forall i, j$ s.t. $i \neq j$. In other words, for complete connectivity schemes, $S_{i} \cap S_{j}$ is always non- 
empty.

The key graph that will be considered in this paper is independent of the communication range which determines the underlying network graph. The reason for this assumption is to have multi hop secure communication between nodes of the network. In other words, we are only concerned with the encryption/decryption of the data sent by the sender and not on the its distance from the receiver. This is particularly useful in cases where the intermediate nodes are not allowed to see the data that is sent by the sender.

In this paper, we analyze the behaviour of deterministic schemes with respect to various performance metrics. We begin by discussing a polynomialbased scheme, which is a simple extension of the line-based scheme [Yuichi Kaji and Matsumoto, 2006]. Polynomials have found use in security system design earlier as well [Vasudevan and Sanyal, 2004], [Vasudevan et al., 2004]. Next, we discuss a scheme based on Symmetric Balanced Incomplete Block Design (SBIBD) [Anderson, 1990] belonging to the complete connectivity category. We provide analytical expressions for resilience and connectivity to compare the three schemes. Motivated by the analysis of the three schemes, we present a general analytical approach for obtaining the resilience and connectivity metrics for a class of key distribution schemes that is not tied to any specific key distribution algorithm. Lastly, we present a design example using our analytical development.

This paper is an extended version of the conference paper [Gupta and Kuri, 2008] and it differs in the following ways: $(i)$ a better justification has been provided for the distribution of the number of keys compromised by the adversary, $(i i)$ a general key allocation scheme is presented along with generalized expressions for security and connectivity measures, $($ iii $)$ a design example is discussed to give insights into the design of a secure system and $(i v)$ proofs for a number lemmas and theorems are also discussed.

Objectives and contributions: In our paper, the focus is on obtaining analytical expressions for connectivity, and resilience when $c$ nodes are captured. We present three deterministic key allocation schemes in Section III, and analyze their performance in the following section.

Section V of the paper presents a general key allocation scheme. The general scheme is described by its properties. The schemes presented earlier are special cases of this general one. Then, this general scheme is analyzed to obtain its connectivity and resilience properties.

The general expressions allow us to show explicitly the trade-off between connectivity and resilience for the three schemes considered. In other words, we show explicitly how improved connectivity leads to decreased resilience.

Further, we use the generalized expressions to understand how user-specified requirements (target connectivity values and resilience values for a specified number of compromised nodes) constrain choices of a specific scheme's parameters. We provide a design example where we start from the user-specified targets, and obtain the ranges of values that the parameters must lie in. The process indicates also when a feasible design meeting the user's requirements does not exist.

The paper is organized as follows. Background is discussed in Section II. In Section III, we discuss polynomial-based schemes and a full connectivity scheme. Analysis of the schemes with comparison is given in Section IV. A generalized analysis for a class of key distribution schemes is discussed in Section $\mathrm{V}$ along with a design example. Section VI concludes the paper.

\section{RELATED WORK}

Key distribution methods in sensor networks are divided into three categories: $(a)$ Probabilistic $(b)$ Deterministic and $(c)$ Hybrid [Camtepe and Yener, 2005]. In case of probabilistic distribution, the key ring for each node is selected randomly from a large pool of keys. Deterministic distribution allocates keys to nodes in a deterministic way to ensure better connectivity. Hybrid schemes use probabilistic methods on deterministic solutions to increase scalability and resilience. In this paper, our focus is on deterministic schemes; however, we survey probabilistic and hybrid schemes for completeness.

The random key pre-distribution scheme was first proposed in [Eschenauer and Gligor, 2002]. In that work, Eschenauer and Gligor suggested a probabilistic solution to the problem of efficient key distribution. In this scheme, each sensor node is assigned a key-ring consisting of $k$ keys chosen at random without replacement from a pool of $\mathcal{P}$ keys. After deployment, two nodes within communication range exchange key-identifiers or challenges to discover common keys. Then, a common key is selected for secure communication. Node pairs without a common key establish a path key through a secure path.

Based on this, several probabilistic schemes with enhanced security features have been suggested. A $q$ composite-random key pre-distribution scheme is proposed by [Chan et al., 2003] which achieves strengthened security under small scale attack while trading off increased vulnerability in the face of a large scale physical attack on network nodes. It then uses multipath key reinforcement scheme to update the commu- 
nication key to a random value after key set-up phase. In [Zhu et al., 2003], a seed-based approach is used for assigning keys to each node. Each key is associated with a unique key identifier or key-id. For any node, a set of key-ids is generated from a common pseudo-random generator with the node identity acting as the seed. The corresponding keys are then stored in the node. This makes it possible for each node to identify the key-ids that another node has, and thereby find if they share any common keys. The seed-based approach reduces the communication burden in sharing key identifiers. [Pietro et al., 2003] uses similar technique for shared key discovery phase.

[Du et al., 2004] gives a scheme where memory requirements can be reduced by utilizing pre-deployment knowledge. In this scheme, knowledge about which nodes are likely to be the neighbours of each sensor node is exploited such that the probability of any two neighbouring nodes sharing a common key is maximized without degrading the other performance metrics, such as security and memory usage.

The scheme in [Du et al., 2003] exhibits a nice threshold property: When the number of compromised nodes is less than the threshold, the probability that any node other than these compromised nodes are affected is close to zero. Their scheme builds on Blom's key predistribution scheme [Blom, 1985] and combines the random key pre-distribution method with it.

In [Liu and Ning, 2003], sensor nodes are deployed in a two dimensional area and each sensor has an expected location that can be predicted. The idea is to have each sensor to share pair-wise keys with its $l$ closest neighbors. In key setup phase, for each sensor node $A$, a unique key $\kappa_{A}$ and $l$ closest neighbors $B_{1}, \ldots$, $B_{l}$ are selected. For each pair $\left(A, B_{i}\right)$, a pair-wise key $\kappa_{A, B_{i}}=\operatorname{PRF}\left(\kappa_{B_{i}} \| a\right)$ is generated, where $a$ is the nodeid of $A$ and $P R F($.$) is the pseudo-random function.$ Node $A$ stores all pair-wise keys, whereas node $B_{i}$ only stores the key $\kappa_{B_{i}}$ and the $P R F($.$) . Thus, each sensor$ uses $2 l+1$ units of memory to store its key-chain.

The key allocation schemes closest in spirit to the ones we discuss in this paper are the deterministic schemes based on combinatorial design techniques [Lee and Stinson, 2005a], [Lee and Stinson, 2005b], [Camtepe and Yener, 2007]. [Lee and Stinson, 2005b] proposes two deterministic schemes: $(i)$ ID-based oneway function scheme, and (ii) multiple space Blom's scheme, where asymmetric key matrices are used instead of symmetric ones. In [Camtepe and Yener, 2007], combinatorial design based pair-wise key predistribution scheme is defined, which is based on the block design techniques in combinatorial design theory.
It employs symmetric and generalized quadrangles design techniques. The scheme uses a finite projective plane of order $n$ (for prime power $n$ ) to generate a symmetric design (or symmetric BIBD) with parameters $\left(n^{2}+n+1, n+1,1\right)$. The design supports $n^{2}+n+1$ nodes and uses key-pool of size $n^{2}+n+1$. It generates $n^{2}+n+1$ key-chains of size $n+1$ where every pair of key-chains has exactly one key in common and every key appears in exactly $n+1$ key-chains.

[Lee and Stinson, 2005b] assumes that the connectivity graph of the nodes satisfies a certain structure. Each node is allocated a secret key, and some other keying material that allows it to compute session keys for those nodes with which it may need to communicate. On the other hand, the schemes that we study do not assume any special structure of the communication graph. Thus, even though [Lee and Stinson, 2005b] allocates keys in a deterministic way, its approach is essentially different from ours.

One of the schemes proposed in [Camtepe and Yener, 2007] is similar to the "complete connectivity" scheme we propose. [Camtepe and Yener, 2007] provides an approximate expression for the fraction of links compromised (which we refer to as "resilience" for short) for a single compromised node. In contrast, we provide analytical expressions for connectivity, as well as resilience when $c$ nodes are compromised, for any $c$. The other schemes in [Camtepe and Yener, 2007] are essentially different; they are based on the "Generalized Quadrangles" approach, while our schemes are based on polynomials over a finite field. Moreover, motivated by the three schemes discussed in Section III, we present a generalized class of key allocation schemes, and obtain analytical expressions for the resilience and connectivity metrics. Further, we use these results to understand how user-specified connectivity and resilience targets indicate the ranges within which the parameters of a specific scheme must lie; this is illustrated by an example in Section V-C.

A part of the discussion in this paper is inspired by a deterministic scheme based on lines over finite plane [Yuichi Kaji and Matsumoto, 2006]. A $p \times p$ grid of keys is chosen, $p$ being prime and keys are distributed to nodes based on the relation $y(x)=a x+b(\bmod p)$. Two nodes share a key if the corresponding lines, over a finite field, intersect each other. If the lines are parallel, then the corresponding nodes do not share a key and needs to undergo pairwise key establishment phase.

An interesting study done by [Xu et al., 2007] has shown that the advantages of the probabilistic approach over the deterministic approach, are not as much as 
people have believed. The work, thus, supports our focus on using deterministic key distribution approach for distributing keys in wireless sensor network.

\section{Three Key Allocation Schemes}

\section{A. Polynomial-based Schemes}

In the polynomial-based scheme, a polynomial of degree $m$ over a residue field $\mathbb{Z}_{p}=\{0,1, \ldots, p-1\}, p$ being a prime, is used to assign keys to a node. The polynomial is represented as: $y(x)=a_{m} x^{m}+a_{m-1} x^{m-1}+$ $\ldots+a_{0}(\bmod p)$, where $y(x), x, a_{m}, a_{m-1}, \ldots, a_{0} \in$ $\mathbb{Z}_{p}$. Two nodes share at least one key between them if the corresponding polynomials intersect at at least one point. Each key is associated with an intersection point in the $p$ $\times p$ grid (Fig. 1). Nodes do not share a key in common

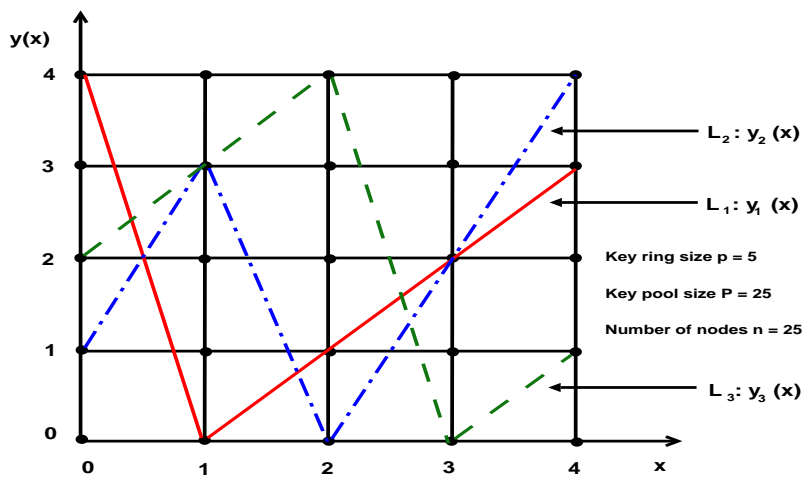

Fig. 1. A $p \times p$ grid containing lines where $p=5$. Each grid point represents a key. A line through the grid represents a node. A node is assigned keys corresponding to the points through which its line passes.

if the corresponding polynomials do not intersect at all.

Since $x$ and $y(x)$ in $y(x)=a_{m} x^{m}+a_{m-1} x^{m-1}+$ $\ldots+a_{0}(\bmod p)$ belong to $\mathbb{Z}_{p}$, the grid size will always be $p \times p$ and the total number of distinct intersection points, corresponding to the points on the grid, will be always $p^{2}$. Each node stores $p$ keys because of the fact that $y(x) \in \mathbb{Z}_{p}$.

We would like to point out here that in general, the expression used for distributing keys to nodes could contain terms both in $x$ and $y$ like $x^{2} y^{3}$ and so on. But we want to restrict our attention to only a specific set of curves which have a particular structure. For example, in the case of polynomial-based scheme when $m=1$, the expression is given as $y(x)=a_{1} x+$ $a_{0}(\bmod p)$. But there exists a more general expression, $a x+b y(\bmod p)=c$ where $y, x, a, b, c \in \mathbb{Z}_{p}$ and $a, b$ are not zero simultaneously, which we will not consider in our analysis.

Since there are $(m+1)$ independent coefficients and each coefficient has $p$ possibilities, the total number of curves possible will be $p^{(m+1)}$ and thus, the number of nodes will be $p^{(m+1)}$. But, in practice, the requirement for the number of nodes will be arbitrary. So, we design the system by choosing a prime power close to the desired value of $n$ but greater than $n$.

Now, we explain the polynomial-based scheme with the help of two schemes: a line-based key distribution scheme [Yuichi Kaji and Matsumoto, 2006] and a degree two polynomial-based key distribution scheme.

1) Scheme 1: Line-based Key distribution scheme:

The intuition behind the scheme, which appeared as a poster paper [Yuichi Kaji and Matsumoto, 2006], is based on the fact that two lines in a plane either intersect at one point or they do not intersect at all [Yuichi Kaji and Matsumoto, 2006]. The authors defined the lines over a residue field $\mathbb{Z}_{p}=\{0,1, \ldots,(p-$ 1) $\}$ where $p$ is a prime. The parameters $a, b$ and the variable $x$ in $y(x)=a x+b(\bmod p)$ take values from the set $\{0,1, \ldots,(p-1)\}$. A particular line $\mathcal{L}$ is thus parallel to $(p-1)$ other lines and intersects with all other lines at exactly one point [Yuichi Kaji and Matsumoto, 2006].

Figure 1 shows an example of such a scheme with $p=5$. For the sake of clarity, only three lines are shown. Lines $\mathcal{L}_{1}, \mathcal{L}_{2}$ and $\mathcal{L}_{3}$ are, respectively, represented by $y_{1}(x)=x+4(\bmod 5), y_{2}(x)=2 x+1(\bmod 5)$ and $y_{3}(x)=x+2(\bmod 5)$. We can see that $\mathcal{L}_{1}$ intersects $\mathcal{L}_{2}$ at exactly one point (having integer coordinates) and is parallel to $\mathcal{L}_{3}$. So, node $\mathcal{N}_{1}$ (corresponding to $\mathcal{L}_{1}$ ) shares a key, obtained by the mapping of the intersection point to a key in the key pool, with $\mathcal{N}_{2}$ (corresponding to $\mathcal{L}_{2}$ ). Also, node $\mathcal{N}_{1}$ does not share a key with node $\mathcal{N}_{3}$ (corresponding to $\mathcal{L}_{3}$ ).

2) Scheme 2: Quadratic Key Distribution Scheme: Yet another example of a scheme belonging to the polynomial-based schemes is a simple extension to the line-based scheme: instead of using a line, use a polynomial of degree two for distributing keys. In the degree two polynomial-based scheme, each node now stores keys corresponding to the points of intersection of a particular curve $y_{j}(x)=a_{j} x^{2}+b_{j} x+c_{j}(\bmod p)$ with the grid points.

Since there exists a one-to-one mapping between curves and nodes, the total number of nodes will be same as the total number of curves. In a degree two polynomial-based scheme, the total number of nodes will be $p^{3}$ as we have three independent coefficients to choose with, each taking $p$ values.

Figure 2 shows an example of such a scheme with $p=5$. Only four curves are shown, for clarity. In the figure, the curves $\mathcal{C}_{1}, \mathcal{C}_{2}, \mathcal{C}_{3}$ and $\mathcal{C}_{4}$ are represented by $y_{1}(x)=0 x^{2}+x+4(\bmod 5), y_{2}(x)=4 x^{2}+$ 


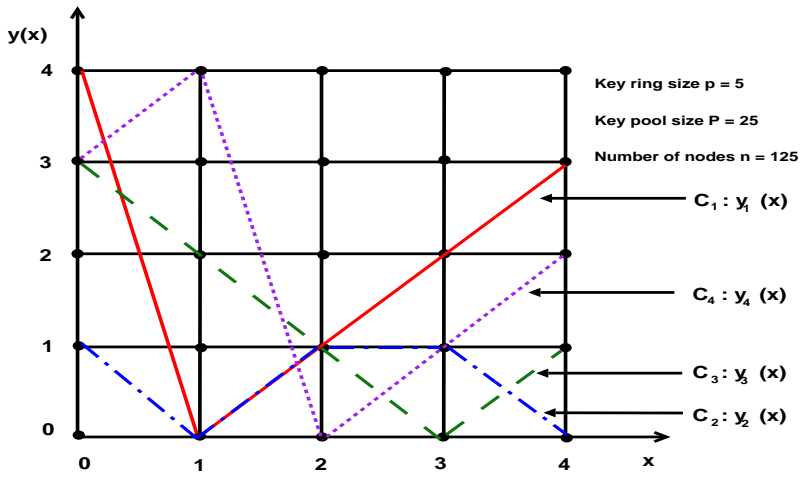

Fig. 2. A $p \times p$ grid for degree two polynomial-based scheme, $p=5$

$0 x+1(\bmod 5), y_{3}(x)=0 x^{2}+4 x+3(\bmod 5)$ and $y_{4}(x)=0 x^{2}+x+3(\bmod 5)$ respectively. We can see that $\mathcal{C}_{1}$ intersects with $\mathcal{C}_{2}$ at exactly two points, intersects with $\mathcal{C}_{3}$ at exactly one point and does not intersects with $\mathcal{C}_{4}$ i.e., is "parallel" to $\mathcal{C}_{4}$. In other words, node $\mathcal{N}_{1}$ (corresponding to $\mathcal{C}_{1}$ ) shares two keys, obtained by the mapping of the intersection points to the keys in the key pool, with $\mathcal{N}_{2}$ (corresponding to $\mathcal{C}_{2}$ ), one key with node $\mathcal{N}_{3}$ ( corresponding to $\mathcal{C}_{3}$ ) and no key with node $\mathcal{N}_{4}$ (corresponding to $\mathcal{C}_{4}$ ).

\section{B. Scheme 3: Complete Connectivity Scheme}

All the schemes discussed till now belong to the first category. We now discuss a scheme belonging to the second category, i.e., a complete connectivity scheme. Here, we will consider $\left|S_{i} \cap S_{j}\right|=2, \forall S_{i}, S_{j} \in S$ and $\forall i, j$ s.t. $i \neq j$.

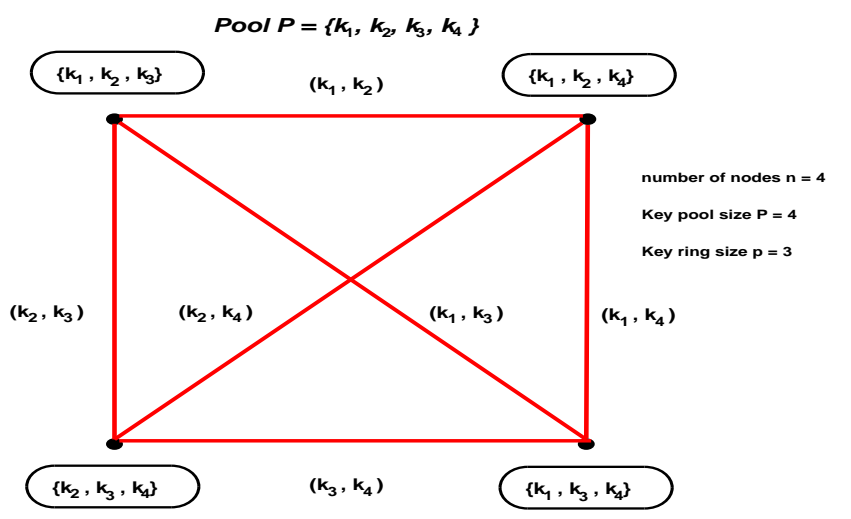

Fig. 3. An example of the key graph for Scheme-3

The scheme is based on Symmetric Balanced Incomplete Block Design (SBIBD) [Anderson, 1990]. A BIBD is an arrangement of $v$ distinct objects into $b$ blocks such that each block contains exactly $k$ distinct objects, each object occurs in exactly $r$ different blocks, and every pair of distinct objects occurs together in exactly $\lambda$ blocks. The design can be expressed as $(v, k, \lambda)$, or equivalently $(v, b, r, k, \lambda)$ where: $\lambda(v-1)=r(k-1)$ and $b k=v r$. A BIBD is called Symmetric BIBD when $b=v$ and therefore $r=k$. The application of SBIBD to sensor network security was first introduced by [Camtepe and Yener, 2007].

In the scheme, $b$ is same as the number of nodes in the network $(n), v$ corresponds to the key pool size $(\mathcal{P}), k$ the number of keys in each node $(p)$ and $r$ corresponds to the number of nodes containing a specific key $(\alpha)$. For the scheme, $\lambda=2$ indicating that a pair of keys is contained in exactly 2 nodes. In other words, we consider all the two-combination of the keys in the pool $\mathcal{P}$ and assign each pair of keys to a link in the network of $n$ nodes. An example of such a scheme is given in Fig. 3 where $n=\mathcal{P}=4$ and $p=3$.

\section{ANALYSIS OF SCHEMES AND COMPARISONS}

\section{A. Notation}

For easy reference, we list in Table I the symbols used in the paper.

\section{TABLE I}

\begin{tabular}{|c|c|}
\hline $\mathcal{P}$ & key pool size \\
\hline$n$ & number of nodes for which the network is being designed \\
\hline$\alpha$ & $\begin{array}{l}\text { number of nodes containing a specific key; same for } \\
\text { all keys }\end{array}$ \\
\hline$p, \beta$ & size of the key ring for each node \\
\hline & number of $j$-key links containing a specific key \\
\hline$\theta$ & $\begin{array}{l}\text { number of disjoint sets into which a key pool is } \\
\text { partitioned }\end{array}$ \\
\hline$\omega$ & number of keys in each partitioned set \\
\hline$m$ & maximum number of keys shared between nodes \\
\hline$\gamma$ & number of no key links per node; a connectivity measure \\
\hline$R_{j}$ & $\begin{array}{l}\text { number of links containing a specific collection of keys } \\
\left\{\kappa_{1}, \kappa_{2}, \ldots, \kappa_{j}\right\}\end{array}$ \\
\hline$K(j)$ & number of $j$-key links in the network $(0 \leq j \leq m)$ \\
\hline & number of nodes captured by the adversary \\
\hline$X_{c}$ & $\begin{array}{l}\text { the random variable denoting the number of keys } \\
\text { compromised when } c \text { nodes are captured }\end{array}$ \\
\hline$p_{c}$ & $\begin{array}{l}\text { the probability that a specific key is contained in at least } \\
\text { one out of } c \text { compromised nodes }\end{array}$ \\
\hline$p_{c}(i)$ & $\begin{array}{l}\text { probability that a particular key is contained in } i \text { out of } c \\
\text { compromised nodes }\end{array}$ \\
\hline
\end{tabular}

\section{B. Definitions}

We use the following definitions for our analysis of various schemes.

Definition 4.1: The security measure is defined as the fraction of the total number of keyed links that are compromised.

By keyed links we mean all those links in the network which are secured by one or more keys. That is, all 
the links in the key graph obtained after the sharedkey discovery phase are referred to as keyed links. A somewhat similar definition is used by authors in [Conti et al., 2007] but for the probabilistic schemes.

Definition 4.2: The connectivity measure is defined as the probability that two randomly chosen sensor nodes share at least one key between them.

By connectivity, we mean the connectivity of the key graph.

We will be frequently using the term j-key links in our subsequent discussions. Let us formally define it here.

Definition 4.3: By a $j$-key link, we mean the tuple $\left(\kappa_{1}, \kappa_{2}, \ldots, \kappa_{j}, N_{i}, N_{l}\right)$ where $N_{i} \& N_{l}, i \neq l$ are the nodes between whom the $j$-key link exists and $\kappa_{1}, \kappa_{2}$, $\ldots, \kappa_{j}$ are the keys that they share in common.

With these definitions in mind, we present the analysis for the three key distribution schemes discussed in the previous section.

\section{Scheme 1: Line-based Key distribution scheme}

Our aim is to find the expected fraction of links compromised by the adversary when $c$ nodes are compromised. To find that, we first need to know the maximum number of keys shared between any pair of nodes and then the number of nodes containing a specific collection of keys. The following lemma puts a restriction on the number of intersection points for any pair of curves.

Lemma 4.1: Let $p$ be a prime, $\mathbb{Z}_{p}=\{0,1, \ldots,(p-$ $1)\}$. Let $a_{0}, a_{1}, \ldots, a_{m}, \quad x \& y(x) \in \mathbb{Z}_{p}$. Let $m<p$. Then, any two curves of degree $m, y(x)=a_{m} x^{m}+$ $a_{m-1} x^{m-1}+\ldots+a_{0}(\bmod p)$, cannot have more than $m$ common intersection points.

Proof: In the Appendix.

Lemma 4.1 shows that for the line-based scheme, there cannot be more than one intersection point for any pair of lines. Since each point of intersection is mapped to a unique key from the key pool $\mathcal{P}$, any pair of nodes either share a key or they do not. Moreover, since each curve is uniquely defined by two points, the total number of curves will be $p^{2}$ as each point can be chosen in $p$ different ways. Thus, we have $p^{2}$ nodes in the network.

Number of nodes containing a specific collection of keys gives insight into the link structure in a key graph. Moreover, it is also required to find an expression for the security measure. In the following lemma, we find the number of curves passing through a given point on the grid.
Lemma 4.2: Let $p$ be a prime, $\mathbb{Z}_{p}=\{0,1, \ldots,(p-$ 1) $\}$. Let $a_{0}, a_{1}, \ldots, a_{m}, x \& y(x) \in \mathbb{Z}_{p}$. Let $m<p$ $\& j \leq m$. Then, the number of curves of degree $m$, $y(x)=a_{m} x^{m}+a_{m-1} x^{m-1}+\ldots+a_{0}(\bmod p)$, passing through $j$ given points on the $p \times p$ grid $\mathbb{Z}_{p}^{2}$ is $p^{(m+1-j)}$.

\section{Proof: In the Appendix.}

Intuitively, since there are $(m+1)$ independent coefficients, the number of curves possible will be $p^{(m+1)}$. Now, if $j$ points on the grid are fixed, then there are $(m+1-j)$ independent points to be chosen to define a curve uniquely. Since each point can be chosen in $p$ ways, there will be $p^{(m+1-j)}$ curves passing through $j$ given points.

Using the above lemma, the number of lines passing through a given point $(j=1)$ will be $p$, as $m=1$ for the line-based scheme. Since each point on the grid is mapped uniquely to a key in the key pool, the number of nodes containing a specific key $\kappa$ will also be $p$.

If we know the expected number of keys compromised by the adversary and also the number of links containing a particular key, then one can easily find the expected number of links compromised by the adversary. Thus, the following lemma gives the distribution of the number of keys compromised by the adversary.

Lemma 4.3: If $n$ is the number of nodes in the network and $\alpha$ is the number of nodes containing a particular key, then for a sufficiently large number of compromised nodes $c$, the number of keys compromised by the adversary $X_{c}$ has a distribution that can be approximated by $\operatorname{Binomial}\left[\mathcal{P},\left(1-p_{c}(0)\right)\right]$ where $p_{c}(0)=$ $\frac{\left(\begin{array}{c}n-\alpha \\ c\end{array}\right)}{\left(\begin{array}{l}n \\ c\end{array}\right)}$ is the probability that a particular key is not compromised.

\section{Proof: In the Appendix.}

From Fig. 4, we see that as the number of nodes compromised by the adversary increases, the distribution of $X_{c}$ moves closer and closer to that of the Binomial distribution. The proof of Lemma 4.3 in the Appendix shows that for tractability, it is necessary to assume that the "state" of a key (defined in the Appendix) is independent of the states of other keys; unless this simplifying approximation is made, the problem becomes too complex to analyze. We see from the four plots that the approximation becomes better and better as the number of compromised nodes $c$ increases.

Since the number of lines passing through a given point is equal to $p(m=1, j=1$ in Lemma 4.2), $\alpha$ $=p$. Thus, the probability that a particular key $\kappa$ is not compromised, when $c$ nodes are captured by the 


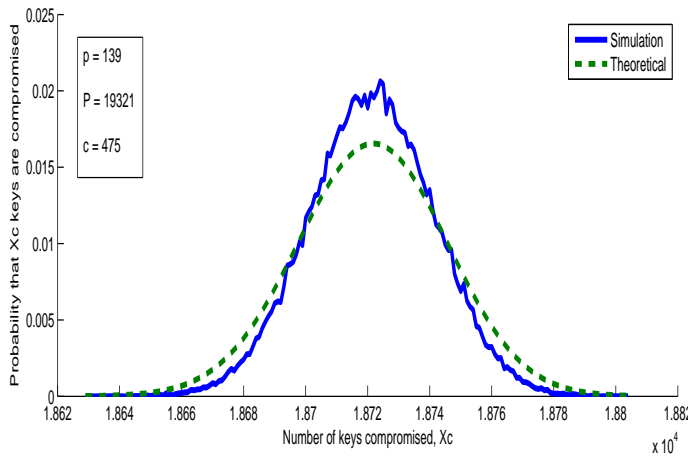

(a) Distribution of $X_{c}$ for $c=475$

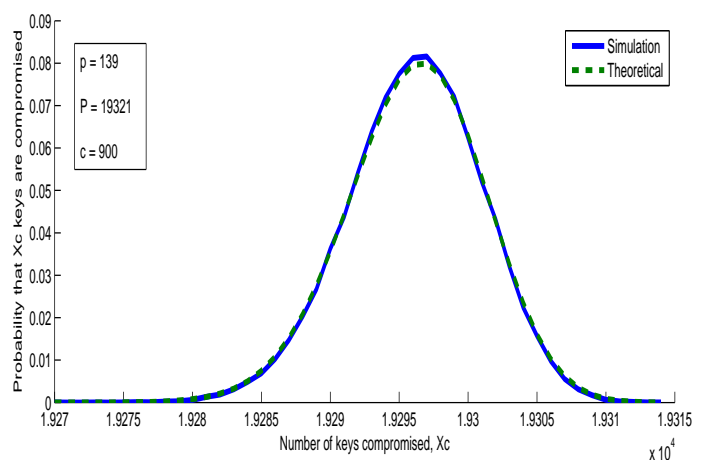

(c) Distribution of $X_{c}$ for $c=900$

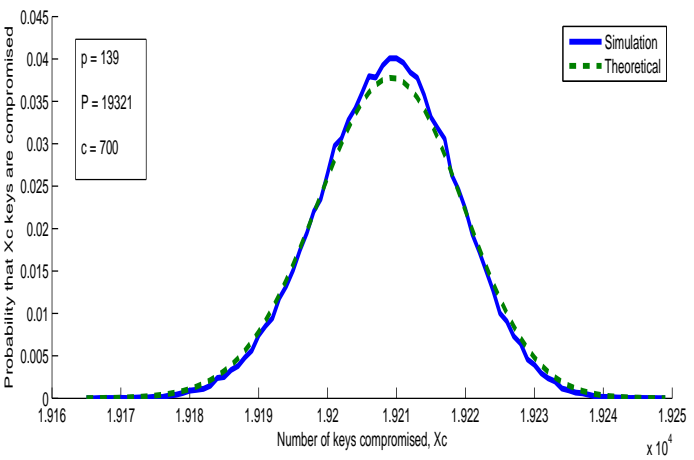

(b) Distribution of $X_{c}$ for $c=700$

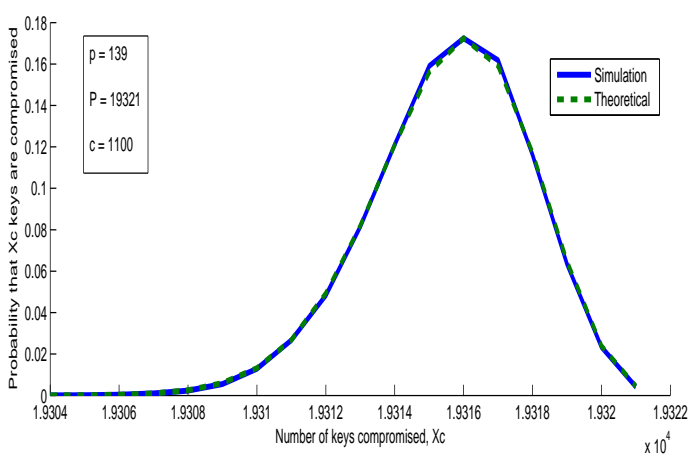

(d) Distribution of $X_{c}$ for $c=1100$

Fig. 4. Theoretical and simulated comparison for distribution of $X_{c}$, the number of keys compromised by the adversary

adversary, will be $p_{c}(0)=\frac{\left(\begin{array}{c}p^{2}-p \\ c\end{array}\right)}{\left(\begin{array}{c}p^{2} \\ c\end{array}\right)}$ and hence, $p_{c}=$ $1-p_{c}(0)$.

Based on the assumed distribution of $X_{c}$, the expected number of keys compromised in the scheme-1 will be $\mathbb{E}\left(X_{c}\right)=p^{2} p_{c}=p^{2}\left(1-\frac{\left(\begin{array}{c}p^{2}-p \\ c\end{array}\right)}{\left(\begin{array}{c}p^{2} \\ c\end{array}\right)}\right)$. Now, since a particular key $\kappa$ is present in $p$ nodes ( $p$ lines pass through a given point), the number of links having the key $\kappa$ will be $\left(\begin{array}{l}p \\ 2\end{array}\right)=\frac{p(p-1)}{2}$. Thus, the expected number of links compromised, when $c$ nodes are captured by the adversary, will be given by the product of the expected number of keys compromised and the number of links containing a particular key $=\frac{p(p-1)}{2} \times p^{2}\left(1-\frac{\left(\begin{array}{c}p^{2}-p \\ c\end{array}\right)}{\left(\begin{array}{c}p^{2} \\ c\end{array}\right)}\right)$ $=\frac{p^{3}(p-1)\left(1-\frac{\left(p^{2}-p\right)}{\left(p^{2}\right)}\right)}{2}$. We note that, in the line-based scheme, a link is secured by using only one key.

Now, let us find the total number of keyed links in a network of $p^{2}$ nodes. Total number of links possible in a network of $p^{2}$ nodes will be $\left(\begin{array}{c}p^{2} \\ 2\end{array}\right)=\frac{p^{2}\left(p^{2}-1\right)}{2}$. Number of no-key links per node (i.e., the links which are not supported by a key) $=(p-1)$, which corresponds to $(p-1)$ parallel lines. Therefore, the total number of nokey links in a network of $p^{2}$ nodes will be $\frac{p^{2}}{2} \times(p-1)=$ $\frac{p^{2}(p-1)}{2}$ where the factor of 2 in the denominator is due to the fact that a secure link $A \rightarrow B$ is same as the secure link $B \rightarrow A$. Once we know the total number of links possible and the total number of no-key links, we can easily calculate the total number of keyed links in the network as $\left(\frac{p^{2}\left(p^{2}-1\right)}{2}-\frac{p^{2}(p-1)}{2}\right)=\frac{p^{3}(p-1)}{2}$.

Thus, the expected fraction of keyed links compromised will be

$$
\begin{aligned}
& \text { Expected fraction of keyed links compromised } \\
& =\frac{\text { Expected number of keyed links compromised }}{\text { Total number of keyed links in the network }} \\
& =1-\frac{\left(\begin{array}{c}
p^{2}-p \\
c
\end{array}\right)}{\left(\begin{array}{c}
p^{2} \\
c
\end{array}\right)}
\end{aligned}
$$

To find the connectivity probability, we need to know the total number of links possible in the network and the total number of keyed links in the network of $p^{2}$ nodes. As found earlier, the total number of links possible is given as $\left(\begin{array}{c}p^{2} \\ 2\end{array}\right)=\frac{p^{2}\left(p^{2}-1\right)}{2}$ and the total number of keyed links $=\frac{p^{3}(p-1)}{2}$. Thus, the connectivity probability is given as

$$
\begin{aligned}
p_{\text {con }} & =\frac{\frac{p^{3}(p-1)}{2}}{\frac{p^{2}\left(p^{2}-1\right)}{2}} \\
& =\frac{p}{p+1}
\end{aligned}
$$




\section{Scheme 2: Quadratic Key Distribution Scheme}

As shown in Lemma 4.1 that the number of intersection points for a polynomial of degree two cannot be more than two, each node will now either share zero, one or two keys with $(n-1)$ other nodes, where $n$ is the total number of nodes supportable by the scheme.

From Lemma 4.2, the number of curves passing through a given point will be $p^{2}$. Thus, each key is now contained in $p^{2}$ nodes. Now, the probability that a particular key is not compromised when $c$ nodes are captured will be $p_{c}^{\prime}=\frac{\left(\frac{p^{3}-p^{2}}{c}\right)}{\left(\begin{array}{c}p^{3} \\ c\end{array}\right)}$. Thus, the expected number of keys compromised is given as $\mathbb{E}\left(X_{c}\right)=p^{2}\left(1-\frac{\left(\begin{array}{c}p^{3}-p^{2} \\ c\end{array}\right)}{\left(\begin{array}{c}p^{3} \\ c\end{array}\right)}\right)$. But this cannot be taken as a measure of security as some of the compromised keys will give rise to one-key links and some will give rise to two-key links. In case of two-key links, it may happen that one key is present in the list of compromised keys but not the other which means that the link is not compromised.

Our aim is to find the expected fraction of the keyed links that are compromised. To achieve this, we divide our analysis into two parts: first we find the expected number of one-key links that are compromised and then we find the expected number of two-key links that are compromised. But before doing that we would first like to find the total number of one-key and two-key links in a network of $p^{3}$ nodes.

Lemma 4.4: For an $m(m<p)$ degree polynomialbased key distribution scheme over $\mathbb{Z}_{p}$ ( $p$ is prime), the total number of $j$-key links (i.e., a link composed of $j$ keys) in a network of $p^{(m+1)}$ nodes is given as

$$
\begin{gathered}
\frac{p^{(m+1)}}{2}\left(p^{(m+1-j)}-1\right)\left(\begin{array}{c}
p \\
j
\end{array}\right)-\frac{p^{(m+1)}}{2} \times \\
\sum_{i=j+1}^{m}(-1)^{(i-j+1)}\left(\begin{array}{c}
i \\
j
\end{array}\right)\left[\left(p^{(m+1-i)}-1\right)\left(\begin{array}{c}
p \\
i
\end{array}\right)\right]
\end{gathered}
$$

Proof: In the Appendix.

Thus, in a network of $p^{3}$ nodes, the total number of one-key links will be $\frac{p^{3}}{2}\left(p^{2}-1\right) p-\frac{p^{3}}{2}\left[2(p-1)\left(\begin{array}{c}p \\ 2\end{array}\right)\right]=$ $p^{4}(p-1)$ and the total number of two-key links will be $\frac{p^{3}}{2}(p-1)\left(\begin{array}{l}p \\ 2\end{array}\right)=\frac{p^{4}(p-1)^{2}}{4}$.

Now, since there are $p^{2}$ keys in all and since a one-key link is composed of only a single key, each one-key link will be repeated $\frac{p^{4}(p-1)}{p^{2}}=p^{2}(p-1)$ times in a network of $p^{3}$ nodes. Thus, the total number of one-key links compromised will be

$$
\begin{aligned}
& \text { Expected number of one-key links compromised } \\
= & \text { Expected number of keys compromised } \\
\times & \text { number of times a particular key is repeated } \\
= & p^{4}(p-1)\left(1-\frac{\left(\begin{array}{c}
p^{3}-p^{2} \\
c
\end{array}\right)}{\left(\begin{array}{c}
p^{3} \\
c
\end{array}\right)}\right)
\end{aligned}
$$

Next, let us find the expected number of two-key links compromised. Since $X_{c}$ is the number of keys compromised and has a distribution Binomial $\left[\mathcal{P}, p_{c}\right]$ (as justified earlier), the expected number of two-key links compromised will be $\mathbb{E}\left(\frac{X_{c}\left(X_{c}-1\right)}{2}\right)$. But $\mathbb{E}\left(\frac{X_{c}\left(X_{c}-1\right)}{2}\right)=$ $\frac{\mathbb{E}\left(X_{c}\right)\left(\mathbb{E}\left(X_{c}\right)-1\right)}{2}+\frac{\operatorname{Var}\left(X_{c}\right)}{2}$ and hence, the expected number of two-key links compromised is given as $\frac{\mathcal{P} p_{c}\left(\mathcal{P} p_{c}-1\right)}{2}+$ $\frac{\mathcal{P} p_{c}\left(1-p_{c}\right)}{2}=\frac{p^{2}\left(p^{2}-1\right)\left(1-\frac{\left(p^{3}-p^{2}\right)}{\left(p_{c}^{3}\right)}\right)^{2}}{2}$.

Now, there are two things we need to consider before arriving at the final expression for the expected number of two-key links that are compromised. First point to be noted is that we cannot have all the two-combination of the keys (i.e., 2-tuple $\left(\kappa_{i}, \kappa_{j}\right)$ where $\left.i \neq j\right)$ captured. This can be explained by referring to Fig. 2. We consider any vertical line along the grid corresponding to a particular value of $x$, say $x=3$. A particular curve, say $\mathcal{C}_{1}: y_{1}(x)=a_{1} x^{2}+b_{1} x+c_{1}(\bmod p)$ will intersect this vertical line at only one point on the grid. Hence, we cannot have two-combination of the keys along this vertical line. Thus, the number of distinct two-key links will be $\frac{p^{2}\left(p^{2}-1\right)}{2}-p \times \frac{p(p-1)}{2}$. The factor of $p$ in the second expression is due to the fact that there are $p$ vertical lines on the grid (since $x \in \mathbb{Z}_{p}$ ). Therefore, the fraction of twocombination of keys that are valid is $\eta=\frac{\frac{p^{2}\left(p^{2}-1\right)}{2}-\frac{p^{2}(p-1)}{2}}{\frac{p^{2}\left(p^{2}-1\right)}{2}}$ $=\left(1-\frac{1}{(p+1)}\right)=\frac{p}{(p+1)}$.

Second point to be noted is that each two-key link is repeated a number of times, a quantity which needs to found. From our previous analysis, the total number of two-key links in a network of $p^{3}$ nodes is given as $\frac{p^{4}(p-1)^{2}}{4}$. Now, the number of distinct twocombination of the keys possible is $\frac{p^{2}\left(p^{2}-1\right)}{2}-p \times \frac{p(p-1)}{2}$, as found earlier. Thus, each two-key link is repeated $\psi=$ $\frac{\text { total number of two-key links }}{\text { number of distinct two-key links }}=\frac{\frac{p^{4}(p-1)^{2}}{4}}{\frac{p^{2}\left(p^{2}-1\right)}{2}-p \times \frac{p(p-1)}{2}}=$ $\frac{p(p-1)}{2}$ times in a network of $p^{3}$ nodes. Our final expression for the expected number of two-key links compromised should include both $\eta$ and $\psi$ factors.

Thus, the expected number of two-key links compromised is given by

$$
\begin{aligned}
& \text { Expected number of two-key links compromised } \\
& =\eta \times \psi \times \frac{p^{2}\left(p^{2}-1\right)\left(1-\frac{\left(\begin{array}{c}
p^{3}-p^{2} \\
c
\end{array}\right)}{\left(\begin{array}{c}
p^{3} \\
c
\end{array}\right)}\right)^{2}}{2}
\end{aligned}
$$




$$
=\left(\frac{p}{(p+1)}\right)\left(\frac{p(p-1)}{2}\right)\left(\frac{p^{2}\left(p^{2}-1\right)\left(1-\frac{\left(\begin{array}{c}
p^{3}-p^{2} \\
c
\end{array}\right)}{\left(\begin{array}{c}
p^{3} \\
c
\end{array}\right)}\right)^{2}}{2}\right)
$$

Now, let us find the total number of keyed links in a network of $p^{3}$ nodes. From our previous analysis, we found that the total number of one-key links $=p^{4}(p-1)$ and the total number of two-key links $=\frac{p^{4}(p-1)^{2}}{4}$ in a network of $p^{3}$ nodes. Thus,

$$
\begin{aligned}
\text { Total number of keyed links } & =p^{4}(p-1)+\frac{p^{4}(p-1)^{2}}{4} \\
& =\frac{p^{4}(p-1)(p+3)}{4}
\end{aligned}
$$

Therefore, the expected fraction of keyed links compromised is given by $((3)+(4)) \div(5)$. That is,

$$
=\frac{4\left(1-\frac{\left(\begin{array}{c}
p^{3}-p^{2} \\
c
\end{array}\right)}{\left(\begin{array}{c}
p^{3} \\
c
\end{array}\right)}\right)+(p-1)\left(1-\frac{\left(\begin{array}{c}
p^{3}-p^{2} \\
c
\end{array}\right)}{\left(\begin{array}{c}
p^{3} \\
c
\end{array}\right)}\right)^{2}}{(p+3)}
$$

To find the connectivity probability for the scheme, we need to know the total number of keyed links and the total number of links possible in the network of $p^{3}$ nodes. The total number of keyed links, as found earlier, is given as $\frac{p^{4}(p-1)(p+3)}{4}$ and the total number of links possible is given as $\frac{p^{3}\left(p^{3}-1\right)}{2}$. Thus, the connectivity probability will be

$$
p_{\text {con }}=\frac{\frac{p^{4}(p-1)(p+3)}{4}}{\frac{p^{3}\left(p^{3}-1\right)}{2}}=\frac{p(p+3)}{2\left(p^{2}+p+1\right)}
$$

\section{E. Scheme 3: Complete Connectivity Scheme}

Since each node stores $p$ keys and each node has $(n-1)$ links, we should have $\frac{p(p-1)}{2}=n-1$. Thus, $n=\mathcal{P}=\frac{p(p-1)}{2}+1$. Now, the probability that a particular key is not compromised when $c$ nodes are captured will be $\frac{\left(\begin{array}{c}n-\alpha \\ c\end{array}\right)}{\left(\begin{array}{l}n \\ c\end{array}\right)}=\frac{\left(\begin{array}{c}n-p \\ c\end{array}\right)}{\left(\begin{array}{l}n \\ c\end{array}\right)}$. Since there are $\mathcal{P}=n$ keys in all, the expected number of keys compromised is given as $\mathbb{E}\left(X_{c}\right)=n\left(1-\frac{\left(\begin{array}{c}n-p \\ c\end{array}\right)}{\left(\begin{array}{l}n \\ c\end{array}\right)}\right)$. Thus, the number of two-key links compromised will be $\mathbb{E}\left(\frac{X_{c}\left(X_{c}-1\right)}{2_{2}}\right)=$ $\frac{\mathbb{E}\left(X_{c}\right)\left(\mathbb{E}\left(X_{c}\right)-1\right)}{2}+\frac{\operatorname{Var}\left(X_{c}\right)}{2}=\frac{n(n-1)\left(1-\frac{\left(\begin{array}{c}n-p \\ c\end{array}\right)}{\left(\begin{array}{c}n \\ c\end{array}\right)}\right)^{2}}{2}$. Now, there are $\frac{n(n-1)}{2}$ two-key links in a network of $n$ nodes. Thus, the expected fraction of keyed links compromised will be

$$
\begin{aligned}
& \text { Expected fraction of keyed links compromised } \\
& =\frac{n(n-1)\left(1-\frac{\left(\begin{array}{c}
n-p \\
c
\end{array}\right)}{\left(\begin{array}{c}
n \\
c
\end{array}\right)}\right)^{2}}{\frac{n(n-1)}{2}}=\left(1-\frac{\left(\begin{array}{c}
n-p \\
c
\end{array}\right)}{\left(\begin{array}{c}
n \\
c
\end{array}\right)}\right)^{2}
\end{aligned}
$$

We note that in this scheme, $p_{\text {con }}=1$.

\section{F. Comparison between the schemes}

A comparison between various schemes for same storage $(p=139)$ is given in Fig. $5(a)$.

Figure 5(b) shows a plot of the probability of connectivity $p_{\text {con }}$ with the number of nodes stored per node $p$. Thus, the quadratic scheme performs better in security but at the expense of connectivity and the line-based \& the SBIBD-based schemes perform better in connectivity at the expense of security. Now, as $p$ becomes large, $p_{c o n}$ for the line-based scheme $\rightarrow 1$ and for the quadratic scheme $\rightarrow \frac{1}{2}$ which is why the curves are almost horizontal for large values of $p$.

We note that even though the storage is same, the degree two polynomial-based scheme accommodates many more nodes than the line-based scheme $\left(p^{3}\right.$ instead of $p^{2}$ ). Because of the much increased number of nodes, the fraction $p_{c o n}$ is smaller.

\section{A Generalized Key Allocation Scheme And ITS ANALYSIS}

The three schemes discussed before suggest a generalized class of key allocation algorithms. We identify the properties that such a generalized scheme must satisfy. In this section, we do not consider any specific key allocation algorithm; rather, our objective is to analyze an entire class of algorithms satisfying certain properties. The three schemes discussed in Section III are specific examples of this class; indeed, they may be viewed as instantiations of the general class of algorithms that we are concerned with.

The generalization presented here is significantly different from the Symmetric BIBD schemes because we allow each node to share at most $m$ keys with every other node in the network, rather than exactly $m$ keys which is the property of BIBD.

\section{A. Generalized Key Distribution Scheme}

Before presenting the analysis for the generalized case, we discuss some of the properties to be satisfied by the generalized class of key distribution algorithm.

1) The key pool set is partitioned into disjoint subsets such that the keys in each subset do not combine to generate a $j$-key link. In other words, a node can select at the most one key from any subset. This takes into account the factor $\eta$, the fraction of key combinations allowed (as defined earlier).

2) Any deterministic key distribution algorithm will support a maximum of $m$ keys that nodes can share among themselves. For polynomial-based schemes, the number of keys shared is at most 


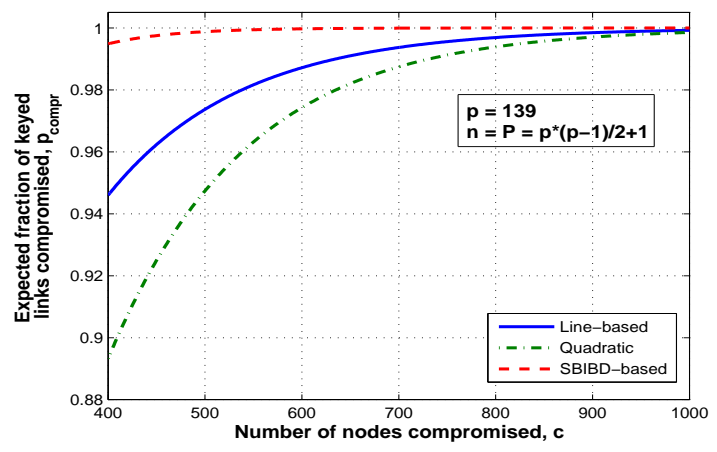

(a) Security comparison between the three schemes

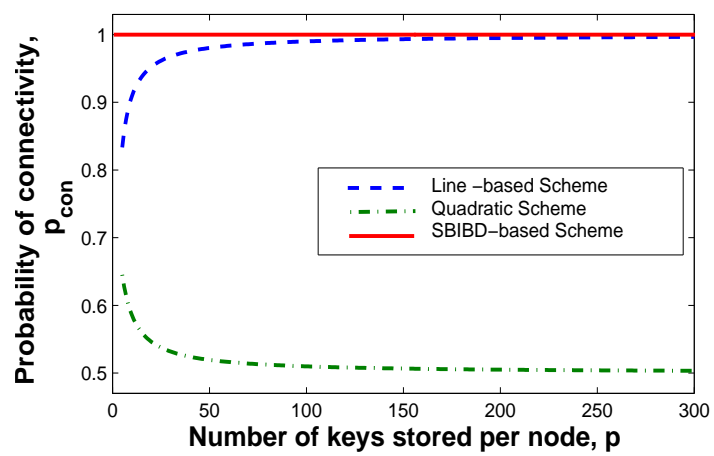

(b) Connectivity comparison

Fig. 5. Among the three schemes, the security measure of the quadratic scheme is best but it comes at the expense of connectivity. The performance of SBIBD based scheme is worst as compared to the polynomial-based schemes.

$m$, while for SBIBD based scheme, the number is exactly $m$.

3) Each key is present in equal number of nodes, as defined by the parameter $\alpha$.

For polynomial-based schemes, property 1 is satisfied by choosing only one point from each vertical line in the grid. For SBIBD, the number of disjoint subsets will be same as the number of elements in the key pool.

The setup for the generalized key allocation scheme is as follows:

1) The key pool set (of size $\mathcal{P}$ ) is partitioned into $\theta$ disjoint subsets, each set being of size $\omega$.

2) Each node is allocated $\beta$ keys from the pool with the following constraints:

a) No more than $m$ keys are shared between any pair of nodes.

b) Exactly $\alpha$ nodes contain a particular key

c) At most one key can be chosen from each disjoint subset. Thus, a particular disjoint subset will be present in $\omega \alpha$ nodes.

d) While allocating keys to the nodes, it is ensured that $\beta \leq \theta$.

3) Number of $j$-key links containing a particular key is the same for all keys. In other words, there does not exist any special key set whose behaviour is different from the rest of the keys' set.

4) An algorithm $\Pi: \Theta \longrightarrow \mathbb{N}$ is defined to distribute keys from the key pool to the nodes in the network where $\Theta$ is the set of subsets of disjoint subsets and $\mathbb{N}$ denotes the set of nodes in the network.

In this paper, we will only concentrate on the analysis of the generalized scheme. Algorithmic aspects will be discussed in our subsequent work.

\section{B. Analysis of the Generalized Scheme}

Our aim is to obtain a generalized expression for security and connectivity measures. Based on the generalized scheme, we will first find the total number of $j$-key links in a network with parameters as $n$ (the number of nodes in the network), $\gamma$ (the number of no-key links per node), $\alpha$ (the number of nodes containing a particular key $\kappa$ ) and $\beta$ (the number of keys per node). Then, we will find the expected number of links compromised by the adversary. Using the two quantities, we will obtain the expression for the resilience measure.

The general expression for the number of $j$-key links $K(j)$ is given as

\section{Lemma 5.1:}

$$
\begin{gathered}
K(j)=\frac{n}{2}\left(\frac{\left(\begin{array}{c}
\theta-j \\
m-j
\end{array}\right)}{\varphi(j)} \omega^{(m-j)}\left\lfloor\frac{\omega(\theta-m)}{\beta-m}\right\rfloor-1\right)\left(\begin{array}{l}
\beta \\
j
\end{array}\right) \\
-\frac{n}{2} \sum_{i=j+1}^{m}(-1)^{(i-j+1)}\left(\begin{array}{c}
i \\
j
\end{array}\right) \times \\
{\left[\left(\frac{\left(\begin{array}{c}
\theta-i \\
m-i
\end{array}\right)}{\varphi(i)} \omega^{(m-i)}\left\lfloor\frac{\omega(\theta-m)}{\beta-m}\right\rfloor-1\right)\left(\begin{array}{c}
\beta \\
i
\end{array}\right)\right]}
\end{gathered}
$$

Proof: In the Appendix.

Here, $\varphi(j)$ is a factor introduced to account for the generation of identical sets of disjoint subsets. The significance of $\varphi(j)$ will be clear if we consider the polynomial-based schemes. In such schemes, each of the disjoint subset contains same type of elements $\{0,1, \ldots,(p-1)\}$, as shown in Fig. 6 for $p=5$. We note that for polynomial-based schemes, any set of $(m+1)$ disjoint subsets results in the same curve as any other set of $(m+1)$ disjoint subsets. Since $j$ sets are already fixed, any set of $(m-j)(<m)$ independent subsets will 


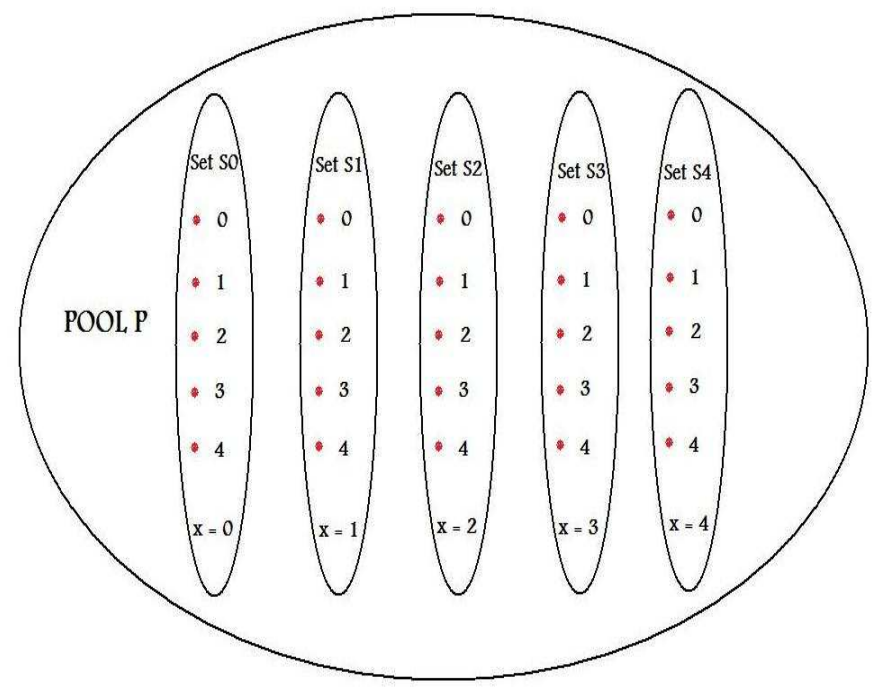

Fig. 6. Partitioning of the key pool set

also result in same distribution of keys as any other set of $(m-j)$ independent subsets. Thus, $\varphi(j)$ for polynomialbased schemes will be

$$
\varphi(j)=\left(\begin{array}{c}
\theta-j \\
m-j
\end{array}\right)
$$

We note that $\varphi(j)$ depends on the specific key distribution algorithm.

Having obtained an expression for the number of $j$ key links, we state the following theorem for the security of a class of key distribution algorithm.

Theorem 5.1: $p_{\text {compr }}=$

$$
\frac{\sum_{j=1}^{m} \frac{(\mathcal{P}-j) ! K(j)}{\mathcal{P} !} T(j)}{\frac{n(n-1-\gamma)}{2}}
$$

where $T(j)=\mathbb{E}\left[X_{c}\left(X_{c}-1\right)\left(X_{c}-2\right) \ldots\left(X_{c}-j+1\right)\right]$ is known as the $j^{\text {th }}$ factorial moment of the distribution of $X_{c}$. Proof: In the Appendix.

Corollary 5.1: For sufficiently large $c$, the security measure is given as

$$
p_{\text {compr }}=\frac{\sum_{j=1}^{m} K(j)\left(1-\frac{\left(\begin{array}{c}
n-\alpha \\
c
\end{array}\right)}{\left(\begin{array}{c}
n \\
c
\end{array}\right)}\right)^{j}}{\frac{n(n-1-\gamma)}{2}}
$$

Proof: In the Appendix.

where $K(j)$ is given by Lemma 5.1.

We observe that the security measure depends on $n$, $\beta, \gamma$ and $\alpha$. We will see in the next section how one can adjust these independent parameters to suit the security requirements.
The connectivity measure $p_{\text {con }}$ can be obtained by making use of $\gamma$. Since the maximum number of links per node can be $(n-1)$, we have:

Theorem 5.2:

$$
p_{\text {con }}=1-\frac{\gamma}{n-1}
$$

We observe that the connectivity measure depends only on $n$ and $\gamma$ and is independent of the other two design parameters: $\alpha$ and $\beta$.

Also, we note that $\theta, \omega$ and $m$ are not system design parameters and can be obtained by solving the following set of three equations:

$$
\sum_{j=1}^{m} K(j)=\frac{n(n-1-\gamma)}{2}
$$

$$
\begin{gathered}
\frac{1}{\mathcal{P}} \sum_{j=1}^{m} j K(j)=\frac{\alpha(\alpha-1)}{2} \\
\omega=\frac{\mathcal{P}}{\theta}
\end{gathered}
$$

Now, when we apply the developed framework, we observe that the three schemes discussed earlier are instantiations of this framework.

Line: $\mathcal{P}=p^{2}, \omega=p, \theta=p, m=1, n=p^{2}, \varphi(j)=$ $\left(\begin{array}{c}p-j \\ m-j\end{array}\right), \alpha=p, \beta=p, \gamma=p-1$

Quad: $\mathcal{P}=p^{2}, \omega=p, \theta=p, m=2, n=p^{3}, \varphi(j)=$ $\left(\begin{array}{c}p-j \\ m-j\end{array}\right), \alpha=p^{2}, \beta=p, \gamma=\frac{(p-1)\left(p^{2}-p+2\right)}{2} ;$

SBIBD: $\mathcal{P}=n, \omega=1, \theta=\mathcal{P}, m=2, n=\frac{s(s-1)}{2}+1, \alpha$ $=s, \beta=s, \gamma=0$.

Equipped with general expressions for $p_{\text {compr }}$ and $p_{\text {con }}$, we make the following observations:

- Since $p_{\text {compr }}=\frac{\sum_{j=1}^{m} \frac{n \beta \delta_{j}}{j \alpha}\left(1-\frac{\left(\begin{array}{c}n-\alpha \\ c\end{array}\right)}{\left(\begin{array}{c}n \\ c\end{array}\right)}\right)^{j}}{\frac{n(n-1-\gamma)}{2}}$ (in the proof of Theorem 5.1), assuming $n, \delta_{j}$ 's (the number of $j$-key links containing a specific key) and $p_{\text {compr }}$ as constants, we observe that there exists a linear relationship between storage and connectivity for a given value of $c$ :

$$
p_{\text {con }}=k \beta
$$

where $k=\frac{\sum_{j=1}^{m} \frac{n \delta_{j}}{j \alpha}\left(1-\frac{\left(\begin{array}{c}n-\alpha \\ c\end{array}\right)}{\left(\begin{array}{c}c \\ j\end{array}\right)}\right)^{j}}{p_{c o m p r} \frac{n(n-1)}{2}}$. The above equation says that, for the same level of security, one can increase connectivity but only at the expense of increase in storage.

- The expression for $p_{\text {compr }}$ for $m=2$ can also be obtained in terms of $n, \alpha, \beta$ and $\gamma$ by solving Eqn. (10) and Eqn. (11) for $K(1)$ and $K(2)$ and then 


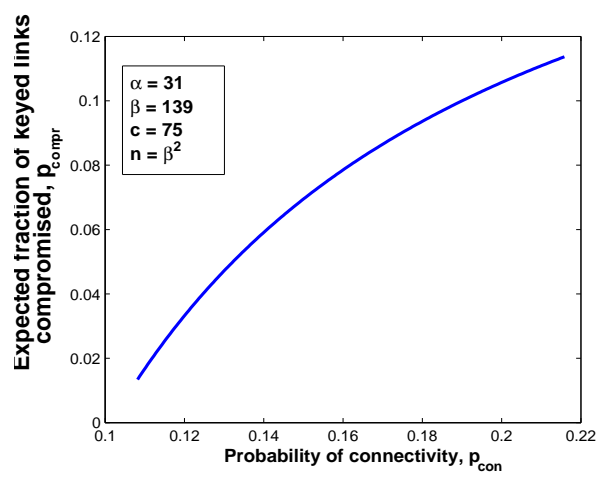

(a) Line-based Scheme

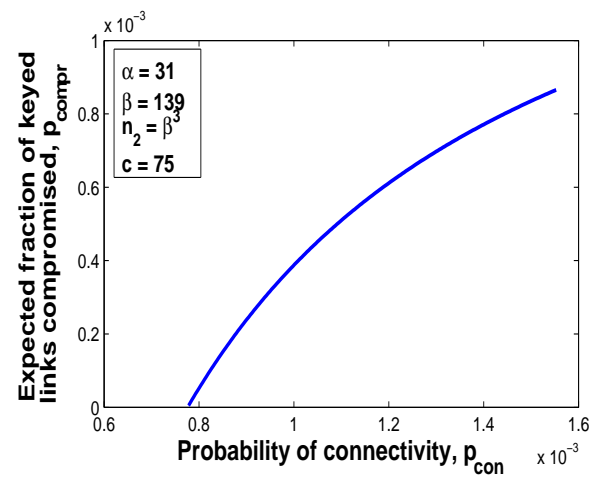

(b) Quadratic Scheme

Fig. 7. Variation of $p_{\text {compr }}$ with respect to $p_{c o n}$; there exists a trade-off between security and connectivity: increasing connectivity decreases security of the system and vice-versa.

substituting in the Theorem 5.1. We note that $\mathcal{P}=$ $\frac{n \beta}{\alpha}$. Thus, we have

$$
\begin{aligned}
p_{\text {compr }} & =\frac{2\left(n(n-1-\gamma)-\frac{n \beta(\alpha-1)}{2}\right)\left(1-\frac{\left(\begin{array}{c}
n-\alpha \\
c
\end{array}\right)}{\left(\begin{array}{c}
n \\
c
\end{array}\right)}\right)}{n(n-1-\gamma)} \\
& +\frac{(n \beta(\alpha-1)-n(n-1-\gamma))\left(1-\frac{\left(\begin{array}{c}
n-\alpha \\
c
\end{array}\right)}{\left(\begin{array}{c}
n \\
c
\end{array}\right)}\right)^{2}}{n(n-1-\gamma)}
\end{aligned}
$$

By expressing $\gamma$ in terms of $p_{c o n}$, we obtain a relation between $p_{\text {compr }}$ and $p_{\text {con }}$. Figure 7 shows plots between the two, for the three schemes. We explicitly show here a widely accepted statement that there exists a trade-off between $p_{\text {compr }}$ and $p_{\text {con }}$. One can achieve better security but at the expense of connectivity and vice-versa.

- Figure 8 shows plots for $p_{\text {compr }}$ with respect to $c$ (the number of nodes compromised by the adversary) for the three schemes, with $\alpha$ being the parameter. The plots show that the system becomes less secure as the number of nodes containing a particular key increases.

\section{Design Example}

Now we consider a design example in which we find appropriate values of the parameters satisfying the system constraints given by the user. For our example, we will consider $m=2$, indicating that the nodes share at most two keys. For this case, we use Eqn. 14 as the expression for $p_{\text {compr }}$. We note here that since $\delta_{1} \geq 0$ and $\delta_{2} \geq 0$ in the proof of the Theorem 5.1, $\alpha$ cannot take all values and is limited by the constraints $\alpha \geq 1+\frac{(n-1-\gamma)}{\beta}$ and $\alpha \leq 1+\frac{2(n-1-\gamma)}{\beta}$. Also, since $\delta_{1}$ and $\delta_{2}$ should be integers, $(n-1-\gamma)$ should be a multiple of $\beta$.

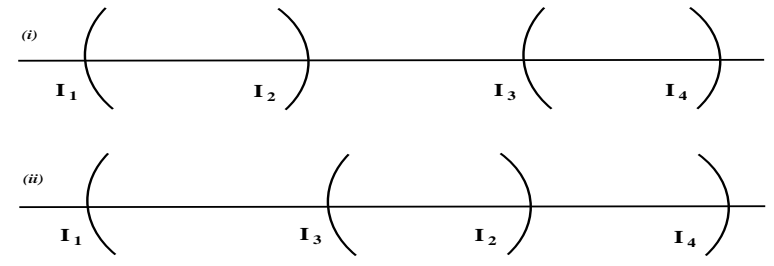

Fig. 9. The two cases: (i) $I_{2}<I_{3}$ and (ii) $I_{2} \geq I_{3}$

To start with, let the number of nodes in the network be $n=n^{\prime}$. In our design, we would like to have $p_{\text {con }}$ to be at least $a$ (i.e., $p_{c o n} \geq a$ ) and the security measure to be not more than $b$ for $c=c^{\prime}$, the number of nodes compromised by the adversary (i.e., $p_{\text {compr }} \leq b$ at $c=$ $c^{\prime}$ ). In other words, we would like to have a system in which the security measure $p_{\text {compr }}$ is always less than $b$, for all values of $c$ less than $c^{\prime}$. Also, let us assume that the storage requirement is $x \leq \beta \leq y$. The reason why one would like to have a minimum storage is because of the linear relationship between $p_{c o n}$ and $\beta$.

From the Theorem 5.2, we see that $\gamma \leq n^{\prime}(1-a)$. Let us assume that $\gamma=r$ where $r \leq n^{\prime}(1-a)$. Now, since $\alpha \geq 1+\frac{(n-1-\gamma)}{\beta}$ and $\alpha \leq 1+\frac{2(n-1-\gamma)}{\beta}$, we have the following four constraints corresponding to the allowed extreme values of the $\beta$.

$$
\begin{array}{ll}
I_{1}: & \alpha \geq 1+\frac{\left(n^{\prime}-1-r\right)}{y} \\
I_{2}: & \alpha \leq 1+\frac{2\left(n^{\prime}-1-r\right)}{y} \\
I_{3}: & \alpha \geq 1+\frac{\left(n^{\prime}-1-r\right)}{x} \\
I_{4}: & \alpha \leq 1+\frac{2\left(n^{\prime}-1-r\right)}{x}
\end{array}
$$

Based on the above constraints, one can have one of the two possibilities: (i) $I_{2}<I_{3}$ and (ii) $I_{2} \geq I_{3}$, as 


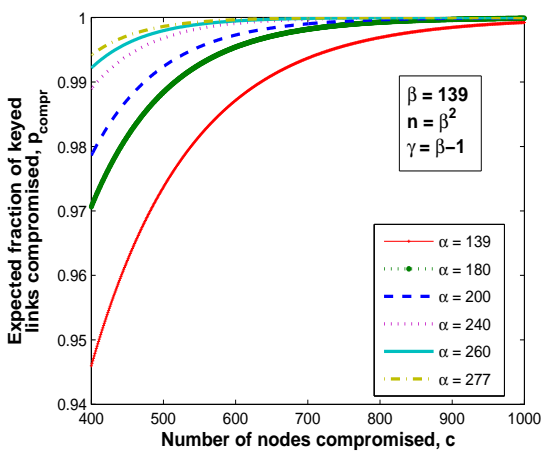

(a) Line-based Scheme

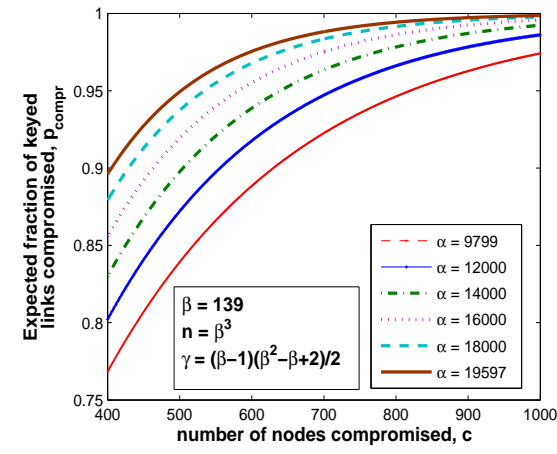

(b) Quadratic Scheme

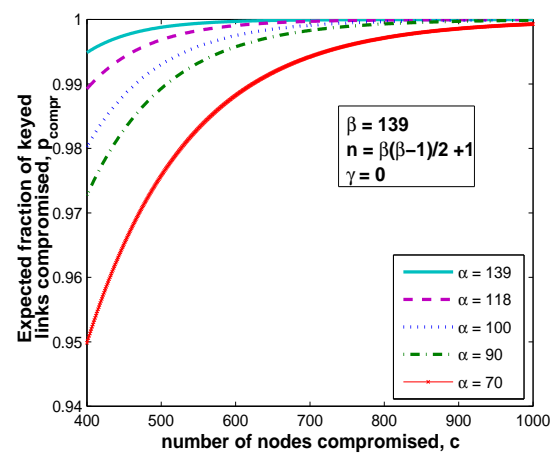

(c) Complete Connectivity Scheme

Fig. 8. Variation of $p_{\text {compr }}$ with respect to $\alpha$; As the number of nodes containing a particular key increases, the security of the system decreases. But $\alpha$ cannot be arbitrarily small as it needs to satisfy the constraints: $\alpha \geq 1+\frac{(n-1-\gamma)}{\beta}$ and $\alpha \leq 1+\frac{2(n-1-\gamma)}{\beta}$.

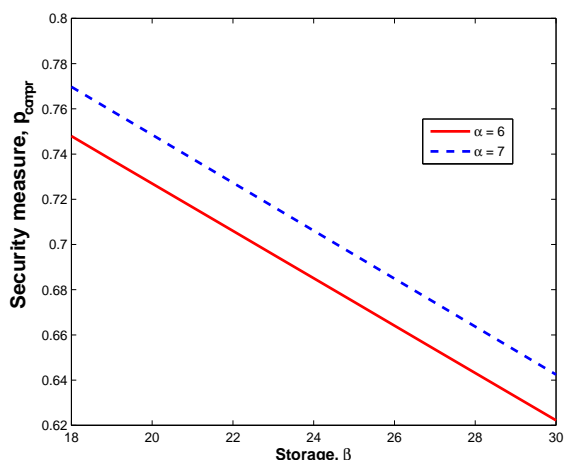

Fig. 10. Security measure $p_{\text {compr }}$ w.r.t. storage $\beta$ with $\alpha=6$ and $\alpha=7$

shown in Fig. 9. In the first case, it is clear that there exists no value of $\alpha$ for which the storage constraint $x \leq \beta \leq y$ is satisfied. Thus, in order to avoid this situation, we should have the second case where $I_{2} \geq I_{3}$ which implies that $y \leq 2 x$. Thus, we have

$$
x \leq \beta \leq y \leq 2 x
$$

The consequence of the above relationship is that if the system designer puts a constraint on the maximum storage value, then there exists a minimum value of the storage below which the design does not exist at all. Now, in order to have more flexibility in choosing the storage value, one needs to squeeze the interval between $I_{3}$ and $I_{2}$ (case (ii) in Fig. 9) which implies a lesser flexibility in choosing values for $\alpha$ (the number of nodes containing a specific key) and thus, a trade-off.

To present a specific design, let us choose specific numerical values of the parameters. Let $n^{\prime}=100, c^{\prime}=$ $20, a=0.9$ and $y=30$. Thus, $r \leq 10$ and $x \geq 15$. Let us assume that $r=9$ and $x=18$. Now, $I_{2}$ will be $\alpha \leq 7$ and $I_{3}$ will be $\alpha \geq 6$. Thus, we have $6 \leq \alpha \leq 7$.
Figure 10 shows a plot of $p_{\text {compr }}$ w.r.t. $\beta$ for the two values of $\alpha$. The plot clearly shows that one cannot achieve $p_{\text {compr }}$ below 0.62 which means that $b>0.62$. Thus, with this design, the best $p_{\text {compr }}$ that one can achieve is $\approx 0.62$ at $c^{\prime}=20$.

Now, let us consider a special case when our design depends only on one parameter. All the three schemes discussed in the previous section belong to this category. We will take scheme-2 as an example. Let $n^{\prime}=125$ and $c^{\prime}=10$. Since $n=\beta^{3}$, the storage will be $\beta=5$. With this value of storage, the other parameters will be $\alpha=$ $\beta^{2}=25$ and $\gamma=\frac{(p-1)\left(p^{2}-p+2\right)}{2}=44$. Using Eqn. (6), the expected fraction of keyed links compromised by the adversary $=0.8584$. In other words, our design should be such that the security measure should be less than 0.8584 for all values of $c$ less than 10 . Now, on simulating the above design, the expected fraction of keyed links compromised comes out to be 0.8563 , thus meeting the user's specifications and validating our analytical expressions.

\section{Conclusion}

The paper discussed about key distribution in wireless sensor networks. We presented three different schemes, each having advantages and disadvantages. A study of these schemes showed that links secured by two keys add to security. The degree two polynomial-based scheme performed better in security but poorly in terms of connectivity. The line-based scheme performed better in connectivity but poorly in terms of security. Finally, the complete connectivity scheme scored perfectly in connectivity and performed variably in terms of security, as compared to the line-based scheme.

In a bottom-up approach for the key distribution procedure, an analysis for a class of key distribution algorithms is first carried out and then, subsequently, 
the algorithm is presented. In this paper, we presented the first part of the procedure whereby we derived generalized expressions for the security and connectivity measures, based on the learning from the three schemes. The expressions clearly showed that in order to design a system, one require four independent parameters: the number of nodes in the network, the number of nokey links per node, the number of nodes containing a particular key and the number of keys per node.

Based on the generalized expressions, we explicitly showed some trade-offs between various design parameters. In particular, we showed that $(i)$ for same security, one can increase connectivity but only at the expense of increase in storage, $(i i)$ a trade-off exists between the security and the connectivity measure and (iii) one can gain in security for less number of nodes compromised but only at the expense of lower security for large number of nodes compromised. Finally, with the help of the generalized expressions, we discussed an example for designing the security system with the given constraints.

The connectivity measure can be used for the energy analysis of a key exchange process, as discussed in [Gupta et al., 2007]. With $p_{\text {con }}$ in hand, a similar energy analysis can be carried out for any key exchange algorithm, thereby enabling an energy cost comparison between various key exchange algorithms.

In this paper, we have only presented an analysis for the generalized key allocation schemes. In our subsequent work, we plan to present a construction for the distribution of keys to nodes based on the analysis and system requirements. Moreover, existence of such an algorithm itself would be an interesting area of exploration.

\section{REFERENCES}

[Akyildiz et al., 2002] Akyildiz, I. F., Su, W., Sankarasubramaniam, Y., and Cayirci, E. (2002). A survey on sensor networks. In IEEE Communications Magazine.

[Anderson, 1990] Anderson, I. (1990). Combinatorial designs: construction methods. Ellis Horwood Limited.

[Batina et al., 2006] Batina, L., Mentens, N., Sakiyama, K., Preneel, B., and Verbauwhede, I. (2006). Low-cost elliptic curve cryptography for wireless sensor networks. In Security and Privacy in Ad-Hoc and Sensor Networks, Third European Workshop, ESAS, volume 4357 of Lecture Notes in Computer Science, pages 6-17. Springer.

[Becher et al., 2006] Becher, A., Benenson, Z., and Dornseif, M. (2006). Tampering with motes: Real-world physical attacks on wireless sensor networks. In J.A. Clark et al. (Eds.): SPC 2006, LNCS 3934, pages 104-118.

[Blom, 1985] Blom, R. (1985). An optimal class of symmetric key generation systems. In Proc. of the EUROCRYPT 84 workshop on Advances in cryptology: theory and application of cryptographic techniques, ISBN: 0-387-16076-0, pages 335-338, New York, NY, USA. Springer-Verlag New York, Inc.
[Bokareva1 et al., 2006] Bokareva1, T., Hu, W., Kanhere, S., Ristic, B., Gordon, N., Bessell, T., Rutten, M., and Jha1, S. (2006). Wireless sensor networks for battlefield surveillance. In Land Warfare Conference, Brisbane.

[Camtepe and Yener, 2007] Camtepe, S. A. and Yener, B. (April, 2007). Combinatorial design of key diistribution mechanisms for wireless sensor networks. IEEE/ACM Transactions On Networking, 15(2).

[Camtepe and Yener, 2005] Camtepe, S. A. and Yener, B. (March 23, 2005). Key distribution mechanisms for wireless sensor networks: A survey. Technical Report TR-05-07, Rensselaer Polytechnic Institute, Computer Science Department. available at http://www.cs.rpi.edu/research/pdf/05-07.pdf.

[Chan et al., 2003] Chan, H., Perrig, A., and Song, D. (2003). Random key predistribution schemes for sensor networks. In Proceedings of IEEE Symposium on Research in Security and Privacy.

[Chong and Kumar, 2003] Chong, C.-Y. and Kumar, S. P. (2003). Sensor networks: Evolution, opportunities, and challenges. In Proceedings of the IEEE, volume 91, No.8.

[Conti et al., 2007] Conti, M., Pietro, R. D., and Mancini, L. V. (2007). Ecce: Enhanced cooperative channel establishment for secure pair-wise communication in wireless sensor networks. In Ad Hoc Networks (Elsevier), volume 5(1): 49-62.

[Culler et al., 2004] Culler, D., Estrin, D., and Srivastava, M. (2004). An overview of sensor networks. In IEEE Computer Society.

[Du et al., 2004] Du, W., Deng, J., Han, Y. S., Chen, S., and Varshney, P. K. (2004). A key management scheme for wireless sensor networks using deployment knowledge. In Proceedings of the IEEE INFOCOM.

[Du et al., 2003] Du, W., Deng, J., Han, Y. S., and Varshney, P. K. (2003). A pairwise key pre-distribution scheme for wireless sensor networks. In Proceedings of the 10th ACM Conference on Computer and Communications Security (CCS).

[Eschenauer and Gligor, 2002] Eschenauer, L. and Gligor, V. D. (2002). A key-management scheme for distributed sensor networks. In Proceedings of the 9th ACM Conference on Computer and Communications Security (CCS).

[Gupta and Kuri, 2008] Gupta, A. and Kuri, J. (2008). Deterministic schemes for key distribution in wireless sensor networks. In Proceedings of the third IEEE/Create-Net/ICST Conference on COMmunication System softWAre and middlewaRE (COMSWARE).

[Gupta et al., 2007] Gupta, A., Nuggehalli, P., and Kuri, J. (2007). An efficient scheme for establishing pair-wise keys for wireless sensor networks. In Proceedings of the first annual workshop on WIreless Systems: Advanced Research and Development (WIS$A R D)$, Bangalore, India.

[Lee and Stinson, 2005a] Lee, J. and Stinson, D. R. (2005a). A combinatorial approach to key predistribution for distributed sensor networks. In IEEE WCNC, pages 1200-1205.

[Lee and Stinson, 2005b] Lee, J. and Stinson, D. R. (2005b). Deterministic key pre-distribution schemes for distributed sensor networks. In ACM Symp. Applied Computing, volume LNCS 3357, pages 294-307.

[Liu and Ning, 2003] Liu, D. and Ning, P. (2003). Location-based pairwise key establishments for relatively static sensor networks. In 2003 ACM Workshop on Security of Ad Hoc and Sensor Networks (SASN03), George W. Johnson Center at George Mason University, Fairfax, VA, USA.

[Pietro et al., 2003] Pietro, R. D., Mancini, L. V., and Mei, A. (2003). Random key assignment for secure wireless sensor networks. In 2003 ACM Workshop on Security of Ad Hoc and Sensor Networks, George W. Johnson Center at George Mason University, Fairfax, VA, USA.

[Szewczyk et al., 2004] Szewczyk, R., Osterweil, E., Polastre, J., Hamilton, M., Mainwaring, A., and Estrin, D. (2004). Habitat 
monitoring with sensor networks. In Communications of the ACM, volume 47, No.6.

[Uhsadel et al., 2007] Uhsadel, L., Poschmann, A., and Paar, C. (2007). Enabling full-size public-key algorithms on 8-bit sensor nodes. In Fourth European Workshop on Security and Privacy in Ad hoc and Sensor Networks - ESAS 2007, Lecture Notes in Computer Science, Cambridge, UK. Springer.

[Vasudevan and Sanyal, 2004] Vasudevan, R. and Sanyal, S. (2004). A Novel Multipath Approach to Security in Mobile Ad Hoc Networks (MANETs). In Proceedings of the International Conference on Computers and Devices for Communication (CODEC'04), pages CAN_0412_CO_F_1-CAN_0412_CO_F_4.

[Vasudevan et al., 2004] Vasudevan, R. A., Abraham, A., Sanyal, S., and Agrawal, D. P. (2004). Jigsaw-based Secure Data Transfer over Computer Networks. In Proceedings of the IEEE International Conference on Information Technology, Coding and Computing (ITCC'04), pages 2-6.

[Wang and Li, 2006] Wang, H. and Li, Q. (2006). Efficient implementation of public key cryptosystems on mote sensors (short paper). In International Conference of Information and Communication Security (ICICS), pages 519-528, Raleigh, NC.

[Xu et al., 2007] Xu, D., Huang, J., Dowskin, J., Chiang, M., and Lee, R. (2007). Reexamining probabilistic vs deterministic key management in mobile ad hoc networks. In IEEE International Symposium on Information Theory (ISIT), Nice, France.

[Yuichi Kaji and Matsumoto, 2006] Yuichi Kaji, H. M. and Matsumoto, R. (2006). Key predistribution schemes for sensor networks using lines and points over a finite geometry. In IEEE SECON(poster paper).

[Zhu et al., 2003] Zhu, S., Xu, S., Setia, S., and Jajodia, S. (2003). Establishing pairwise keys for secure communication in ad hoc networks: a probabilistic approach. In Proceedings of the 11th IEEE International Conference on Network Protocols (ICNP).

\section{APPENDIX}

\section{PROOFS OF LEMMAS AND THEOREMS}

Proof: [Lemma 4.1] To prove the lemma, we invoke Lagrange's Theorem for finite fields. It states that

"For $m \geq 0$, let $x_{0}, x_{1}, \ldots, x_{m}$ be $m+1$ distinct elements of a finite field $\mathcal{F}$ and let $y_{0}, y_{1}, \ldots, y_{m}$ be $m+1$ arbitrary elements of $\mathcal{F}$. Then there exists exactly one polynomial $p(x)$ with coefficients in $\mathcal{F}$ and of degree $\leq m$ such that $p\left(x_{j}\right)=y_{j}$ for $j=0,1, \ldots, m$. The polynomial is given by "

$$
p(x)=\sum_{j=0}^{m} b_{j} \prod_{k=0, k \neq j}^{m}\left(x_{j}-x_{k}\right)^{-1}\left(x-x_{k}\right)
$$

Since $p$ is prime, Lagrange's theorem holds. Thus, we cannot have more than $m$ intersection points otherwise the polynomial will not be unique.

Proof: [Lemma 4.2] Let the $j$ given points be $A_{1}=$ $\left(x_{1}, y\left(x_{1}\right)\right), A_{2}=\left(x_{2}, y\left(x_{2}\right)\right), \ldots, A_{j}=\left(x_{j}, y\left(x_{j}\right)\right)$. Now, in order to uniquely define a curve $\mathcal{C}: y(x)$, we require $(m+1-j)$ more grid points $\left(x_{i}, y\left(x_{i}\right)\right), i=$ $j+1, j+2, \ldots,(m+1-j)$, other than $A_{1}, A_{2}, \ldots, A_{j}$.
This is because we require $(m+1)$ points to uniquely construct an $m$ degree polynomial by using Lagrange's method. But, for each value of $x_{i}$, there are $\left(\begin{array}{l}p \\ 1\end{array}\right)$ ways in which $y\left(x_{i}\right)$ could be obtained (points corresponding to the vertical line on the grid, for a given value of $x=x_{i}$ [see Fig. 1]). As we require $(m+1-j)$ points (apart from $A_{1}, A_{2}, \ldots, A_{j}$ ) to construct the curve $\mathcal{C}$, the number of ways in which such a curve could be constructed = $p^{(m+1-j)}$.

We note that even though there are $\left(\begin{array}{c}p-j \\ m+1-j\end{array}\right)$ ways of selecting $(m+1-j)$ points, only one selection is actually sufficient. This is because we are counting the same curve $\left(\begin{array}{c}p-j \\ m+1-j\end{array}\right)$ times. Let us see why this is so. Given a set $\mathbb{Y}=\left\{y_{j+1}, y_{j+2}, \ldots, y_{m+1}\right\}$ of values of $y(x)$ corresponding to a particular set $\mathbb{X}=\left\{x_{j+1}, x_{j+2}, \ldots, x_{m+1}\right\}$ of values of $x$, there exists $(p-(m+1))$ values of $y(x)$ $\mathbb{Y}^{\prime}=\left\{y_{m+2}, y_{m+3}, \ldots, y_{p}\right\}$ corresponding to values of $x$ other than the $(m+1)$ values already used to generate the unique curve. Now, let us define a set $\mathbb{Y}^{\prime \prime}=\mathbb{Y}+$ $\mathbb{Y}^{\prime}=\left\{y_{j+1}, y_{j+2}, \ldots, y_{m+1}, y_{m+2}, y_{m+3}, \ldots, y_{p}\right\}$ where these values are corresponding to the values of $x$ in the set $\mathbb{X}^{\prime \prime}=\left\{x_{j+1}, x_{j+2}, \ldots, x_{p}\right\}$. Choosing any of the $(m+1-j)$ values of $x$ from the set $\mathbb{X}^{\prime \prime}$ will result in the same curve. Thus, each curve is counted $\left(\begin{array}{c}p-j \\ m+1-j\end{array}\right)$ times.

Hence, $p^{(m+1-j)}$ curves pass through $j$ given points $A_{1}, A_{2}, \ldots, A_{j}$.

Proof: [Lemma 4.3] When an adversary captures $c$ nodes, each of the $\mathcal{P}$ keys in the key pool are in any one of the following $\alpha+1$ states:

- $A_{0}$ : a key in this state will not be present in the compromised pool of keys

- $A_{1}$ : a key in this state will be present only once in the compromised pool of keys

- $A_{2}$ : a key in this state will be present only twice in the compromised pool of keys

- $A_{\alpha}$ : a key in this state will be present $\alpha$ times in the compromised pool of keys

We note that here $\alpha$ represents the number of nodes containing a specific key, say $\kappa$.

Let $Y_{i}, i=0,1, \ldots, \alpha$ be the random variable denoting the number of keys in a particular state $A_{i}$. Now, when $c$ nodes are compromised, the total number of keys compromised (including the repeated keys) $=p c$ where $p$ is the key ring size for each node. Thus, for any $c$, the random variables $Y_{i}$ 's should satisfy the condition

$$
1 * Y_{1}+2 * Y_{2}+\ldots+\alpha * Y_{\alpha}=p c
$$

where $\alpha$ is the number of nodes containing a specific key. 
Now, we are interested in the number of distinct keys compromised which is given by the random variable

$$
X_{c}=Y_{1}+Y_{2}+\ldots+Y_{\alpha}
$$

We note that, for any value of $c$, the following relationship holds:

$$
\mathcal{P}=Y_{0}+Y_{1}+Y_{2}+\ldots+Y_{\alpha}
$$

which implies that

$$
X_{c}=\mathcal{P}-Y_{0}
$$

where $Y_{0}$ and $X_{c}$ are random variables and $\mathcal{P}$ is a positive constant (the key pool size). Thus, by knowing the distribution of $Y_{0}$, one can deduce the distribution of $X_{c}$ and vice-versa.

Thus, the distribution of $X_{c}$ can be found by using the expression in Eqn. (21). Let $p_{c}(i), i=0,1, \ldots, \alpha$, denote the probability that a particular key $\kappa$ is in state $A_{i}$. Since $\alpha$ nodes contain the key $\kappa$, the number of ways in which $i$ nodes can be chosen from these $\alpha$ nodes will be $\left(\begin{array}{c}\alpha \\ i\end{array}\right)$. So, $p_{c}(i)$ is given as

$$
p_{c}(i)=\frac{\left(\begin{array}{c}
\alpha \\
i
\end{array}\right)\left(\begin{array}{c}
n-\alpha \\
c-i
\end{array}\right)}{\left(\begin{array}{l}
n \\
c
\end{array}\right)}
$$

It can be seen that

$$
\sum_{i=0}^{\min (c, \alpha)} p_{c}(i)=1
$$

Now, let us find the probability that $Y_{i}=k$ which is nothing but the probability that $k$ out of $\mathcal{P}$ keys are in state $A_{i}$. Now, assuming that each key takes a particular state independent of other keys, it can be easily seen that the probability $\operatorname{Pr}\left(Y_{i}=k\right)$ is given as

$$
\operatorname{Pr}\left(Y_{i}=k\right)=\left(\begin{array}{l}
\mathcal{P} \\
k
\end{array}\right) p_{c}(i)^{k}\left(1-p_{c}(i)\right)^{\mathcal{P}-k}
$$

For $i=0$, we have

$$
\operatorname{Pr}\left(Y_{0}=k\right)=\left(\begin{array}{l}
\mathcal{P} \\
k
\end{array}\right) p_{c}(0)^{k}\left(1-p_{c}(0)\right)^{\mathcal{P}-k}
$$

Thus, the probability distribution of $X_{c}$ is given by

$$
\begin{aligned}
\operatorname{Pr}\left(X_{c}=k\right) & =\operatorname{Pr}\left(Y_{0}=\mathcal{P}-k\right) \\
& =\left(\begin{array}{l}
\mathcal{P} \\
k
\end{array}\right) p_{c}(0)^{\mathcal{P}-k}\left(1-p_{c}(0)\right)^{k}
\end{aligned}
$$

where $p_{c}(0)=\frac{\left(\begin{array}{c}n-\alpha \\ c\end{array}\right)}{\left(\begin{array}{l}n \\ c\end{array}\right)}$.

Proof: [Lemma 4.4] Let $\mathbb{N}$ denote the node set corresponding to the nodes in the network. Let $\mathbb{I}_{j}^{[i k]}$ be the set of $j$ key sets each corresponding to the intersection points between a pair of polynomials of degree $m, y_{i}(x)=a_{m} x^{m}+a_{m-1} x^{m-1}+\ldots+a_{0}$ and $y_{k}(x)=b_{m} x^{m}+b_{m-1} x^{m-1}+\ldots+b_{0}$, having exactly $j$ points of intersection. Let us also define the following sets:

$\mathbb{X}_{j}^{a}=$ the set of all the sets of the form: $\left\{\left\{A_{i}, A_{k}\right\}, \mathbb{I}_{j}^{[i k]}\right\}$ s.t. $A_{i}, A_{k} \in \mathbb{N}$ share exactly j keys i.e., the set of all j-key links\}

$$
\begin{aligned}
\mathbb{Y}_{j}^{[i k]}= & \{\text { the set of all the sets of the form: } \\
& \left\{\mathbb{I}_{j}^{[i k]}, \kappa_{1}, \ldots, \kappa_{l}\right\} \text { s.t. the nodes } A_{i}, A_{k} \\
& \text { share exactly }(j+l) \text { keys containing } j \\
& \text { keys from the set } \mathbb{I}_{j}^{[i k]}, \forall l=0,1,2, \ldots, \\
& (m-j)\}
\end{aligned}
$$

$$
\begin{aligned}
\mathbb{Z}_{j}^{[i k]}= & \{\text { the set of all the sets of the form: } \\
& \left\{\mathbb{I}_{j}^{[i k]}, \kappa_{1}, \ldots, \kappa_{l}\right\} \text { s.t. the nodes } A_{i}, A_{k} \\
& \text { share exactly }(j+1) \text { keys containing } j \\
& \text { keys from the set } \mathbb{I}_{j}^{[i k]}, \forall l=1,2, \ldots, \\
& (m-j)\}
\end{aligned}
$$

$$
\begin{aligned}
& \mathbb{X}_{j}^{\prime}=\{\text { the set of all the sets of the form: } \\
& \left\{\left\{A_{i}, A_{k}\right\}, \mathbb{J}_{(.)}^{[i k]}\right\} \text { s.t. for each } A_{i}, A_{k} \in \mathbb{N} \text {, } \\
& \text { all the key sets } \mathbb{J}_{(.)}^{[i k]} \text { belong to the } \\
& \text { corresponding set } \left.\mathbb{Y}_{j}^{[i k]}\right\} \\
& \mathbb{X}_{j}=\mathbb{X}_{j}^{\prime}+x_{j}
\end{aligned}
$$

where $x_{j}$ is the set accounting for over counting.

$$
\begin{aligned}
\mathbb{W}_{j}= & \{\text { the set of all the sets of the form: } \\
& \left\{\left\{A_{i}, A_{k}\right\}, \mathbb{J}_{(.)}^{[i k]}\right\} \text { s.t. for each } A_{i}, A_{k} \in \mathbb{N}, \\
& \text { all the key sets } \mathbb{J}_{(.)}^{[i k]} \text { belong to the } \\
& \text { corresponding set } \left.\mathbb{Z}_{j}^{[i k]}\right\}
\end{aligned}
$$

In other words, $\mathbb{X}_{j}^{a}$ is the set in which a node pair $\left(A_{i}, A_{k}\right)$ results in an actual $j$-key link $\mathbb{I}_{j}^{[i k]}$ with all such node pairs from the set $\mathbb{N}$. $\mathbb{Y}_{j}^{[i k]}$ is the set of set of keys in which each key set corresponds to an l-key link with $j$-keys belonging to the set $\mathbb{I}_{j}^{[i k]}(l \geq j)$. $\mathbb{Z}_{j}^{[i k]}$ is the same set as the set $\mathbb{Y}_{j}^{[i k]}$ but with the condition that $l>j . \mathbb{X}_{j}^{\prime}$ is the set in which each node pair $\left(A_{i}, A_{k}\right)$ shares $l(\geq j)$ keys, with $j$ keys belonging to the set $\mathbb{I}_{j}^{[i k]}$. $\mathbb{W}_{j}$ is the same set as the set $\mathbb{X}_{j}$ but with the condition that $l>j$.

Now, to find the number of elements in the set $\mathbb{X}_{j}$, we first find the number of $j$-key links per node. Let us fix $j(\leq m)$ keys $\kappa_{1}, \kappa_{2}, \ldots, \kappa_{j}$, out of $p$ (key ring size) 
keys of a node, say $A$. The number of nodes, other than node $A$, having the same $j$ keys $\kappa_{1}, \kappa_{2}, \ldots, \kappa_{j}$ will be $\left(p^{(m+1-j)}-1\right)$ (using Lemma 4.2). But the $j$ keys $\kappa_{1}, \kappa_{2}, \ldots, \kappa_{j}$ could be chosen in $\left(\begin{array}{l}p \\ j\end{array}\right)$ ways. Thus, the total number of $j$-key links per node will be $\left(p^{(m+1-j)}-\right.$ 1) $\times\left(\begin{array}{l}p \\ j\end{array}\right)$. Since there are $p^{(m+1)}$ nodes in all and since link from $B \rightarrow C$ is same as the link $C \rightarrow B$, we have $\left|\mathbb{X}_{j}\right|=\frac{p^{(m+1)}\left(p^{(m+1-j)}-1\right)\left(\begin{array}{l}p \\ j\end{array}\right)}{2}$. But this cannot be taken as the actual number of $j$-key links in the network. This is because $\left|\mathbb{X}_{j}\right|$ also includes $j$-key links from the keys constituting a $(j+1)$-key link, a $(j+2)$-key link, ... a $m$-key link.

Let us represent the number of generated $j$-key links from the keys of an $i$-key link by $\left|\mathbb{G}_{i}^{[j]}\right|$ where $i=$ $(j+1),(j+2), \ldots, m$. Then, from the definitions of $\mathbb{X}_{j}$ and $\mathbb{X}_{j}^{a}$, it is clear that $\left|\mathbb{X}_{m}^{a}\right|=\left|\mathbb{X}_{m}\right|\left(\mathbb{W}_{m}=\phi\right)$ for a degree- $m$ polynomial-based scheme. Now,

$$
\begin{aligned}
\left|\mathbb{X}_{m-1}^{a}\right| & =\left|\mathbb{X}_{m-1}\right|-\left|\mathbb{W}_{m-1}\right| \\
\left|\mathbb{X}_{m-2}^{a}\right| & =\left|\mathbb{X}_{m-2}\right|-\left|\mathbb{W}_{m-2}\right| \\
\vdots & \\
\left|\mathbb{X}_{j}^{a}\right| & =\left|\mathbb{X}_{j}\right|-\left|\mathbb{W}_{j}\right|
\end{aligned}
$$

But $\left|\mathbb{W}_{m-1}\right|,\left|\mathbb{W}_{m-2}\right|, \ldots,\left|\mathbb{W}_{j}\right|$ can be written as

$$
\begin{aligned}
\left|\mathbb{W}_{m-1}\right| & =\left|\mathbb{G}_{m}^{[m-1]}\right| \\
\left|\mathbb{W}_{m-2}\right| & =\left|\mathbb{G}_{m-1}^{[m-2]}\right|+\left|\mathbb{G}_{m}^{[m-2]}\right| \\
\vdots & \\
\left|\mathbb{W}_{j}\right| & =\left|\mathbb{G}_{j+1}^{[j]}\right|+\left|\mathbb{G}_{j+2}^{[j]}\right|+\ldots+\left|\mathbb{G}_{m}^{[j]}\right|
\end{aligned}
$$

Thus, we have

$$
\left|\mathbb{X}_{m-1}^{a}\right|=\left|\mathbb{X}_{m-1}\right|-\left|\mathbb{G}_{m}^{[m-1]}\right|
$$

Similarly, we have

$$
\begin{gathered}
\left|\mathbb{X}_{m-2}^{a}\right|=\left|\mathbb{X}_{m-2}\right|-\left|\mathbb{G}_{m-1}^{[m-2]}\right|-\left|\mathbb{G}_{m}^{[m-2]}\right| \\
\vdots \\
\left|\mathbb{X}_{j}^{a}\right|=\left|\mathbb{X}_{j}\right|-\left|\mathbb{G}_{j+1}^{[j]}\right|-\left|\mathbb{G}_{j+1}^{[j]}\right| \ldots-\left|\mathbb{G}_{m}^{[j]}\right|
\end{gathered}
$$

Let us, now, find $\left|\mathbb{G}_{i}^{[j]}\right|$, for $i=j+1, j+2, \ldots, m$. The number of $j$-key links contributed by the keys of an $i$-key link in $\mathbb{X}_{i}^{a}$ will be $\left(\begin{array}{l}i \\ j\end{array}\right)$, where $i=j+1, j+2, \ldots, m$. Thus, we have

$$
\left|\mathbb{G}_{i}^{[j]}\right|=\left(\begin{array}{l}
i \\
j
\end{array}\right)\left|\mathbb{X}_{i}^{a}\right|
$$

Therefore, the number of $j$-key links in a degree- $m$ polynomial-based scheme will be given as

$$
\begin{aligned}
\left|\mathbb{X}_{j}^{a}\right| & =\left|\mathbb{X}_{j}\right|-\left(\begin{array}{c}
j+1 \\
j
\end{array}\right)\left|\mathbb{X}_{j+1}^{a}\right|-\left(\begin{array}{c}
j+2 \\
j
\end{array}\right)\left|\mathbb{X}_{j+2}^{a}\right|-\ldots \\
& -\left(\begin{array}{c}
m \\
j
\end{array}\right)\left|\mathbb{X}_{m}^{a}\right|
\end{aligned}
$$

Rewriting the above equation in terms of $l(=m-j)$, we have

$$
\begin{aligned}
\left|\mathbb{X}_{m-l}^{a}\right| & =\left|\mathbb{X}_{m-l}\right|-\left(\begin{array}{c}
m-l+1 \\
m-l
\end{array}\right)\left|\mathbb{X}_{m-l+1}^{a}\right| \\
& -\left(\begin{array}{c}
m-l+2 \\
m-l
\end{array}\right)\left|\mathbb{X}_{m-l+2}^{a}\right|-\ldots \\
& -\left(\begin{array}{c}
m \\
m-l
\end{array}\right)\left|\mathbb{X}_{m}^{a}\right|
\end{aligned}
$$

Now, let us prove the formula for $l=1$ and $l=2$. For $l=1$, we have

$$
\begin{aligned}
\left|\mathbb{X}_{m}^{a}\right| & =\left|\mathbb{X}_{m}\right| \\
& =\frac{p^{(m+1)}\left(p^{(m+1-m)}-1\right)\left(\begin{array}{c}
p \\
m
\end{array}\right)}{2}
\end{aligned}
$$

For $l=2$, we have

$$
\begin{aligned}
\left|\mathbb{X}_{m-1}^{a}\right| & =\left|\mathbb{X}_{m-1}\right|-\left(\begin{array}{c}
m \\
m-1
\end{array}\right)\left|\mathbb{X}_{m}^{a}\right| \\
& =\frac{p^{(m+1)}\left(p^{(m+1-(m-1))}-1\right)\left(\begin{array}{c}
p \\
m-1
\end{array}\right)}{2} \\
& -\left(\begin{array}{c}
m \\
m-1
\end{array}\right)\left(\frac{p^{(m+1)}\left(p^{(m+1-m)}-1\right)\left(\begin{array}{c}
p \\
m
\end{array}\right)}{2}\right) \\
& =\frac{p^{(m+1)}}{2}\left(p^{(m+1-(m-1))}-1\right)\left(\begin{array}{c}
p \\
m-1
\end{array}\right) \\
& -\frac{p^{(m+1)}}{2}\left(\begin{array}{c}
m \\
m-1
\end{array}\right)\left[\left(p^{(m+1-m)}-1\right)\left(\begin{array}{c}
p \\
m
\end{array}\right)\right]
\end{aligned}
$$

The same expression can be obtained by substituting $j=$ $(m-1)$ in the formula to be proved.

Let us assume it to hold for $l=k$. That is, $\left|\mathbb{X}_{m-k}^{a}\right|$ is given as

$$
\begin{aligned}
\left|\mathbb{X}_{m-k}^{a}\right|= & \frac{p^{(m+1)}}{2} \sum_{i=m-k}^{m}(-1)^{(i-(m-k)+2)}\left(\begin{array}{c}
i \\
m-k
\end{array}\right) \times \\
& {\left[\left(p^{(m+1-i)}-1\right)\left(\begin{array}{c}
p \\
i
\end{array}\right)\right] }
\end{aligned}
$$

Now, let us prove it for $l=k+1$. From equation (33), 
we have

$$
\begin{aligned}
& \left|\mathbb{X}_{m-(k+1)}^{a}\right|=\left|\mathbb{X}_{m-(k+1)}\right|-\left(\begin{array}{c}
m-(k+1)+1 \\
m-(k+1)
\end{array}\right) \times \\
& \left|\mathbb{X}_{m-(k+1)+1}^{a}\right|-\left(\begin{array}{c}
m-(k+1)+2 \\
m-(k+1)
\end{array}\right) \times \\
& \left|\mathbb{x}_{m-(k+1)+2}^{a}\right|-\ldots \\
& -\left(\begin{array}{c}
m-(k+1)+(k+1) \\
m-(k+1)
\end{array}\right)\left|\mathbb{x}_{m}^{a}\right| \\
& =\left|\mathbb{X}_{m-(k+1)}\right|-\sum_{l=0}^{k}\left(\begin{array}{c}
(m-k)+l \\
(m-k)-1
\end{array}\right)\left|\mathbb{X}_{(m-k)+l}^{a}\right| \\
& =\left|\mathbb{X}_{m-(k+1)}\right|-\sum_{l=0}^{k}\left[\left(\begin{array}{c}
(m-k)+l \\
(m-k)-1
\end{array}\right) \times\right. \\
& \frac{p^{(m+1)}}{2} \sum_{i=m-k+l}^{m}(-1)^{(i-(m-k+l)+2)}\left(\begin{array}{c}
i \\
m-k+l
\end{array}\right) \times \\
& {\left[\left(p^{(m+1-i)}-1\right)\left(\begin{array}{l}
p \\
i
\end{array}\right)\right]} \\
& =\left|\mathbb{X}_{m-(k+1)}\right|-\frac{p^{(m+1)}}{2} \sum_{l=0}^{k} \sum_{i=m-k+l}^{(m-k+l)+(k-l)} \\
& (-1)^{(i-(m-k+l)+2)}\left(\begin{array}{c}
(m-k)+l \\
(m-k)-1
\end{array}\right)\left(\begin{array}{c}
i \\
m-k+l
\end{array}\right) \times \\
& {\left[\left(p^{(m+1-i)}-1\right)\left(\begin{array}{l}
p \\
i
\end{array}\right)\right]}
\end{aligned}
$$

Now, we consider only the summation factor. Writing for each value of $l$, we have

$$
\begin{aligned}
l=0: \quad & (-1)^{2} \frac{(m-k) !}{1 !(m-k-1) ! 0 !)}\left[\left(p^{(k+1)}-1\right)\left(\begin{array}{c}
p \\
m-k
\end{array}\right)\right] \\
l=1: & \left((-1)^{1+2} \frac{(m-k+1) !}{1 !(m-k-1) ! 1 !)}+(-1)^{2} \frac{(m-k+1) !}{2 !(m-k-1) ! 0 !)}\right) \times \\
& {\left[\left(p^{((k+1)+1)}-1\right)\left(\begin{array}{c}
p \\
m-k+l
\end{array}\right)\right] }
\end{aligned}
$$

$l=k \quad: \quad\left(\sum_{r=0}^{k}(-1)^{r+2} \frac{(m-k+k) !}{(r+1) !(m-k-1) !(l-r) !}\right)\left(p^{(k+l)}-1\right)\left(\begin{array}{c}p \\ m-k+l\end{array}\right)$

Thus, $\left|\mathbb{X}_{m-(k+1)}^{a}\right|$ is given as

$$
\begin{aligned}
\left|\mathbb{X}_{m-(k+1)}^{a}\right|= & \left|\mathbb{x}_{m-(k+1)}\right|-\frac{p^{(m+1)}}{2} \sum_{i=m-k}^{(m-k)+k} \\
& \left(\sum_{r=0}^{i-(m-k)}(-1)^{r+2} \frac{i !}{(r+1) !(m-k-1) !(i-(m-k)-r) !}\right) \\
& \times\left[\left(p^{(m+1-i)}-1\right)\left(\begin{array}{l}
p \\
i
\end{array}\right)\right] \\
= & \left|\mathbb{x}_{m-(k+1)}\right|-\frac{p^{(m+1)}}{2} \sum_{i=m-k}^{(m-k)+k} \frac{i !}{(m-k-1) !} \times \\
& \left(\sum_{r=0}^{i-(m-k)} \frac{(-1)^{r+2}}{(r+1) !(i-(m-k)-r) !}\right) \times \\
& {\left[\left(p^{(m+1-i)}-1\right)\left(\begin{array}{l}
p \\
i
\end{array}\right)\right] }
\end{aligned}
$$

Now, we consider the following expression

$$
\begin{aligned}
\sum_{r=0}^{a} \frac{(-1)^{r+2}}{(r+1) !(a-r) !} & =\frac{1}{(a+1) !} \sum_{r=0}^{a}(-1)^{r+2}\left(\begin{array}{l}
a+1 \\
r+1
\end{array}\right) \\
& =\frac{1}{(a+1) !}
\end{aligned}
$$

Using the above identity, we have

$$
\begin{aligned}
\left|\mathbb{X}_{m-(k+1)}^{a}\right|= & \left|\mathbb{x}_{m-(k+1)}\right|-\frac{p^{(m+1)}}{2} \sum_{i=m-k}^{(m-k)+k} \\
& \frac{i !}{(m-k-1) !(i-(m-k)+1) !}\left[\left(p^{(m+1-i)}-1\right)\left(\begin{array}{l}
p \\
i
\end{array}\right)\right]
\end{aligned}
$$

Using the expression $\left|\mathbb{X}_{j}\right|=\frac{p^{(m+1)}\left(p^{(m+1-j)}-1\right)\left(\begin{array}{l}p \\ j\end{array}\right)}{2}$, we observe that the formula also holds for $l=k+1$. Hence, it holds for all $l<m$.

Proof: [Lemma 5.1] In case of the polynomial based schemes, the expression derived for the number of $j$-key links in a network is given by Lemma 4.4. Re-writing the above expression in terms of the parameters defined above, the number of $j$-key links present in a network of $n$ nodes is given as

$$
\begin{aligned}
K(j) & =\frac{n}{2}\left((f(j)-1)\left(\begin{array}{c}
\beta \\
j
\end{array}\right)\right) \\
& -\frac{n}{2} \sum_{i=j+1}^{m}(-1)^{(i-j+1)}\left(\begin{array}{c}
i \\
j
\end{array}\right)\left[(f(i)-1)\left(\begin{array}{c}
\beta \\
i
\end{array}\right)\right]
\end{aligned}
$$

where $f(j)$ is defined as the number of nodes containing a specific collection of keys $\left\{\kappa_{1}, \kappa_{2}, \ldots, \kappa_{j}\right\}$.

Now, let us try to find the most general expression for $f(j)$. For this, let us partition the pool of keys into $\theta$ disjoint sets, each containing $\frac{\mathcal{P}}{\theta}$ keys $\left(\mathcal{P}=\frac{n \beta}{\alpha}\right.$ is the size of the key pool). Each of these sets has the property that the keys within each set do not combine to generate a link. Thus, a particular node can pick just one key per set.

Since we limit ourselves to a maximum of $m$-key links, $f(m)$ can be found by fixing $m$ out of $\theta$ sets. Then, the number of nodes having same $m$-tuple of keys will be equal to the number of sets (of size $(\beta-m)$ ) into which $(\mathcal{P}-m)$ keys can be divided. Thus, we have

$$
f(m)=\left\lfloor\frac{\omega(\theta-m)}{\beta-m}\right\rfloor
$$

where $\omega=\frac{\mathcal{P}}{\theta}$.

Now, to find the expression for $f(j)$ for $j<m$, we fix $j$ out of $\theta$ sets. The $(m-j)$ sets can be chosen from $(\theta-j)$ sets in $\left(\begin{array}{c}\theta-j \\ m-j\end{array}\right)$ ways. Since there are $\omega$ keys in each of the $(m-j)$ sets, we have

$$
f(j)=\left(\begin{array}{c}
\theta-j \\
m-j
\end{array}\right) \omega^{(m-j)}\left\lfloor\frac{\omega(\theta-m)}{\beta-m}\right\rfloor
$$

Thus, the general expression for the number of $j$-key links $K(j)$ is given as

$$
\begin{aligned}
K(j)= & \frac{n}{2}\left(\frac{\left(\begin{array}{c}
\theta-j \\
m-j
\end{array}\right)}{\varphi(j)} \omega^{(m-j)}\left\lfloor\frac{\omega(\theta-m)}{\beta-m}\right\rfloor-1\right)\left(\begin{array}{l}
\beta \\
j
\end{array}\right) \\
- & \frac{n}{2} \sum_{i=j+1}^{m}(-1)^{(i-j+1)}\left(\begin{array}{c}
i \\
j
\end{array}\right) \times \\
& {\left[\left(\frac{\left(\begin{array}{c}
\theta-i \\
m-i
\end{array}\right)}{\varphi(i)} \omega^{(m-i)}\left\lfloor\frac{\omega(\theta-m)}{\beta-m}\right\rfloor-1\right)\left(\begin{array}{c}
\beta \\
i
\end{array}\right)\right] }
\end{aligned}
$$

where $\varphi(j)$ is a factor introduced to account for the generation of identical sets of disjoint subsets. This 
factor is introduced to nullify the extra counting which could be introduced by the factor $\left(\begin{array}{c}\theta-j \\ m-j\end{array}\right)$.

Proof: [Theorem 5.1] Let $R_{j}$ be the number of times a particular collection of $j$ keys $\left\{\kappa_{1}, \kappa_{2}, \ldots, \kappa_{j}\right\}$ is repeated in the network and $\eta_{j}$ be the fraction of $j$-key links allowed by the scheme (depends on the number of disjoint sets into which the pool of keys is divided). Then, the security measure is given as

$$
p_{\text {compr }}=\frac{\sum_{j=1}^{m} \eta_{j} R_{j} \mathbb{E}\left[\left(\begin{array}{c}
X_{c} \\
j
\end{array}\right)\right]}{\frac{n(n-1-\gamma)}{2}}
$$

Let us find the expression for each of the $j$ factors in the above equation. Expanding $\mathbb{E}\left[\left(\begin{array}{c}X_{c} \\ j\end{array}\right)\right]$, we have

$$
\begin{aligned}
\mathbb{E}\left[\left(\begin{array}{c}
X_{c} \\
j
\end{array}\right)\right] & =\frac{1}{j !} \mathbb{E}\left[X_{c}\left(X_{c}-1\right)\left(X_{c}-2\right) \ldots\left(X_{c}-j+1\right)\right] \\
& =\frac{T(j)}{j !}
\end{aligned}
$$

where $T(j)=\mathbb{E}\left[X_{c}\left(X_{c}-1\right)\left(X_{c}-2\right) \ldots\left(X_{c}-j+1\right)\right]$ is known as the $j^{\text {th }}$ factorial moment of the distribution of $X_{c}$.

Now, if $\delta_{j}$ denotes the number of $j$-key links containing a particular key $\kappa$, allowed by the scheme, then we have

$$
\eta_{j}=\frac{\delta_{j}}{R_{j}\left(\begin{array}{c}
\mathcal{P}-1 \\
j-1
\end{array}\right)}
$$

Thus, $p_{\text {compr }}$ will be

$$
p_{\text {compr }}=\frac{\sum_{j=1}^{m} \frac{(\mathcal{P}-j) ! \delta_{j}}{(\mathcal{P}-1) !} \frac{T(j)}{j}}{\frac{n(n-1-\gamma)}{2}}
$$

What remains to be found is an expression for $\delta_{j}$ in terms of the system design parameters. Since a specific key is present in $\alpha$ nodes, the number of links among themselves will be $\frac{\alpha(\alpha-1)}{2}$. Thus, we have the relation

$$
\sum_{j=1}^{m} \delta_{j}=\frac{\alpha(\alpha-1)}{2}
$$

Now, since $K(j)$ is the number of $j$-key links, the number of distinct $j$-keys links will be given as $\frac{K(j)}{R_{j}}$. But the number of distinct $j$-links is also given by $\left(\begin{array}{l}\theta \\ j\end{array}\right) \omega^{j}$. Thus, we have

$$
\frac{K(j)}{R_{j}}=\left(\begin{array}{l}
\theta \\
j
\end{array}\right) \omega^{j}
$$

or $R_{j}$ will be

$$
R_{j}=\frac{K(j)}{\left(\begin{array}{c}
\theta \\
j
\end{array}\right) \omega^{j}}
$$

Also, we have

$$
\begin{aligned}
\delta_{j} & =R_{j}\left(\begin{array}{l}
\theta-1 \\
j-1
\end{array}\right) \omega^{(j-1)} \\
& =\frac{K(j) j}{\theta \omega}=\frac{K(j) j}{\mathcal{P}}
\end{aligned}
$$

Thus, the security measure is given as

$$
p_{\text {compr }}=\frac{\sum_{j=1}^{m} \frac{\frac{K(j) j}{\mathcal{P}}}{\left(\begin{array}{c}
\mathcal{P}-1 \\
j-1
\end{array}\right)} \mathbb{E}\left[\left(\begin{array}{c}
X_{c} \\
j
\end{array}\right)\right]}{\frac{n(n-1-\gamma)}{2}}
$$

To check the sanity of the above equation, substituting $X_{c}$ by $\mathcal{P}$, we have

$$
\begin{aligned}
p_{\text {compr }} & =\frac{\sum_{j=1}^{m} \frac{\frac{K(j) j}{\mathcal{P}}}{\left(\begin{array}{c}
\mathcal{P}-1 \\
j-1
\end{array}\right)}\left(\begin{array}{l}
\mathcal{P} \\
j
\end{array}\right)}{\frac{n(n-1-\gamma)}{2}} \\
& =\frac{\sum_{j=1}^{m} K(j)}{\frac{n(n-1-\gamma)}{2}}=1
\end{aligned}
$$

Substituting various values in the Eqn. 46, we have

$$
p_{\text {compr }}=\frac{\sum_{j=1}^{m} \frac{(\mathcal{P}-j) ! K(j)}{\mathcal{P} !} T(j)}{\frac{n(n-1-\gamma)}{2}}
$$

where $K(j)$ denotes the number of $j$-key links in a network of $n$ nodes and is given by Lemma 5.1.

Proof: [Corollary 5.1] As discussed earlier, the probability $p_{c}(0)$ that the $c$ compromised nodes does not contain a specific key $\kappa$ is given as

$$
p_{c}(0)=\frac{\left(\begin{array}{c}
n-\alpha \\
c
\end{array}\right)}{\left(\begin{array}{l}
n \\
c
\end{array}\right)}
$$

where $\alpha$ is the number of nodes containing a specific key $\kappa$. Thus, the probability $p_{c}$ that a particular key is compromised is given as

$$
p_{c}=1-p_{c}(0)=1-\frac{\left(\begin{array}{c}
n-\alpha \\
c
\end{array}\right)}{\left(\begin{array}{c}
n \\
c
\end{array}\right)}
$$

Now, since the probability distribution of $X_{c}$ is approximated by Binomial $\left[\mathcal{P}, p_{c}\right]$ in Lemma $4.3, T(j)$ will be nothing but the $j^{\text {th }}$ factorial moment for a binomial distribution $\operatorname{Binomial}[n, p]$ and is given as

$$
T(j)=\frac{n !}{(n-j) !} p^{j}
$$

Thus, we have

$$
\begin{aligned}
\mathbb{E}\left[\left(\begin{array}{c}
X_{c} \\
j
\end{array}\right)\right] & =\frac{1}{j !} T(j) \\
& =\left(\begin{array}{c}
\mathcal{P} \\
j
\end{array}\right) p_{c}{ }^{j}
\end{aligned}
$$


Thus, the security measure is given as

$$
p_{\text {compr }}=\frac{\sum_{j=1}^{m} K(j)\left(1-p_{c}(0)\right)^{j}}{\frac{n(n-1-\gamma)}{2}}
$$

